\title{
CONJUGACY CLASSES IN REDUCTIVE GROUPS AND TWO-SIDED CELLS
}

\author{
G. LUSZTIG \\ Department of Mathematics, M.I.T., Cambridge, MA 02139, USA. \\ E-mail: gyuri@math.mit.edu \\ \|\|\|\|

\begin{abstract}
Let $G^{\prime}$ be a connected reductive group over the complex numbers. We show that the set of conjugacy classes of elements of $G^{\prime}$ with semisimple part of finite order is in natural bijection with the set of two-sided cells associated to a certain algebra.
\end{abstract}

\section{Introduction}

0.1. Let $G^{\prime}$ be a connected reductive group over C. In [4] it was shown that the set of unipotent conjugacy classes of $G^{\prime}$ is in natural bijection with the set of two-sided cells coming from an affine Hecke algebra associated to $G$, a reductive group of type dual to that of $G^{\prime}$. In this paper we extend this to a bijection between the set of all conjugacy classes of elements of $G^{\prime}$ with semisimple part of finite order and the set of two-sided cells associated to a certain algebra $H$ containing the affine Hecke algebra, see Theorem 2.6. The algebra $H$ is an affine analogue of an algebra considered in [7, §31] which, on the one hand, is a modified form of an algebra considered by Mars and Springer [9] in their approach to the theory of character sheaves and, on the other hand, as shown in [8, §34], is closely related to the algebra studied by Yokonuma in [10]. Most of the paper is concerned with showing that the notion of two-sided cell can be defined for $H$.

Received November 9, 2017 and in revised form February 1, 2018.

AMS Subject Classification: 20G99.

Key words and phrases: Conjugacy class, affine Weyl group, two-sided cell.

Supported by NSF grant DMS-1566618. 
0.2. Notation. Let $\mathcal{R}$ be a commutative ring with 1 and let $A$ be an associative $\mathcal{R}$-algebra with a given $\mathcal{R}$-basis $\left\{b_{i} ; i \in I\right\}$ where $I \neq \emptyset$. For $K \subset I$ we write $[K]=\sum_{i \in K} \mathcal{R} b_{i} \subset A$. For $i, i^{\prime} \in I$ we write $i^{\prime} \sim_{\text {left }} i$ if for any $K \subset I$ such that $[K]$ is a left ideal of $A$ we have $i \in K$ if and only if $i^{\prime} \in K$. This is an equivalence relation on $I$; the equivalence classes are called the left cells of $I$. Replacing "left" by "right" we obtain an equivalence relation $\sim_{\text {right }}$ on $I$; the equivalence classes are called the right cells of $I$. Replacing "left" by "two-sided" we obtain an equivalence relation $\sim$ on $I$; the equivalence classes are called the two-sided cells of $I$.

By an affine Weyl group we mean a finite product of irreducible affine Weyl groups. In particular, the group with one element is an affine Weyl group.

Let $\mathcal{A}=\mathbf{Z}\left[v, v^{-1}\right]$ where $v$ is an indeterminate.

\section{Two-sided Cells}

1.1. Let $\mathbf{k}, \mathbf{k}^{\prime}$ be algebraically closed fields. Let $G$ be a connected reductive group over $\mathbf{k}$ with a fixed Borel subgroup $\mathbf{B}$ and a fixed maximal torus $\mathbf{T} \subset \mathbf{B}$. Let $\rho=\operatorname{dim} \mathbf{T}, \nu=\operatorname{dim} G / \mathbf{B}$.

Let $G^{\prime}$ be a connected reductive group over $\mathbf{k}^{\prime}$ of type dual to that of $G$ with a fixed Borel subgroup $\mathbf{B}^{\prime}$ and a fixed maximal torus $\mathbf{T}^{\prime} \subset \mathbf{B}^{\prime}$.

Let $\underline{L}$ (resp. $\underline{L}^{\prime}$ ) be the lattice of one parameter subgroups $\mathbf{k}^{*} \rightarrow \mathbf{T}$ (resp. $\left.\mathbf{k}^{\prime *} \rightarrow \mathbf{T}^{\prime}\right)$. We assume that $\underline{L}$ is also the group of characters $\mathbf{T}^{\prime} \rightarrow \mathbf{k}^{\prime *}$ and that $\underline{L}^{\prime}$ is also the group of characters $\mathbf{T} \rightarrow \mathbf{k}^{*}$. We assume that the obvious nondegenerate pairing $\langle\rangle:, \underline{L} \times \underline{L}^{\prime} \rightarrow \mathbf{Z}$ defined in terms of $\mathbf{T}$ is the same as that defined in terms of $\mathbf{T}^{\prime}$. Let $\underline{R} \subset \underline{L}$ (resp. $\underline{R}^{\prime} \subset \underline{L}^{\prime}$ ) be the set of coroots of $G$ (resp. $G^{\prime}$ ) with respect to $\mathbf{T}$ (resp. $\mathbf{T}^{\prime}$ ); we assume that $\underline{R}$ (resp. $\underline{R}^{\prime}$ ) is also the set of roots of $G^{\prime}$ (resp. of $G$ ) with respect to $\mathbf{T}^{\prime}$ (resp. $\mathbf{T})$. We also assume that the canonical bijection $\underline{R} \leftrightarrow \underline{R}^{\prime}$ defined in terms of $G$ is the same as that defined in terms of $G^{\prime}$; we denote it by $h \leftrightarrow h^{\prime}$. Let $\left\{h_{i} ; i \in \underline{I}\right\} \subset \underline{R}$ be the set of simple roots of $G^{\prime}$ determined by $\mathbf{B}^{\prime}$; we assume that $\left\{h_{i}^{\prime} ; i \in \underline{I}\right\} \subset \underline{R}^{\prime}$ is the set of simple roots of $G$ determined by $\mathbf{B}$.

Let $\underline{V}=\mathbf{Q} \otimes \underline{L}, \underline{V}^{\prime}=\mathbf{Q} \otimes \underline{L}^{\prime}$; now $\langle$,$\rangle extends to a nondegenerate bilinear$ pairing $\langle\rangle:, \underline{V} \times \underline{V}^{\prime} \rightarrow \mathbf{Q}$. Let $V$ (resp. $V^{\prime}$ ) be a $\mathbf{Q}$-vector space containing 
$\underline{V}\left(\right.$ resp. $\left.\underline{V}^{\prime}\right)$ as a subspace and let $\langle\rangle:, V \times V^{\prime} \rightarrow \mathbf{Q}$ be a nondegenerate bilinear pairing extending $\langle\rangle:, \underline{V} \times \underline{V^{\prime}} \rightarrow \mathbf{Q}$.

We shall consider two cases (we will refer to them as case A and case B).

(A) We have $V=\underline{V}, V^{\prime}=\underline{V^{\prime}}$. We set $L=\underline{L}, L^{\prime}=\underline{L^{\prime}}$.

(B) We have $V=\underline{V} \oplus \mathbf{Q} c, V^{\prime}=\underline{V}^{\prime} \oplus \mathbf{Q} c^{\prime}$ where $c, c^{\prime}$ are vectors such that $\left\langle c, c^{\prime}\right\rangle=1,\left\langle c, \underline{V}^{\prime}\right\rangle=0,\left\langle\underline{V}, c^{\prime}\right\rangle=0$. We set $L=\underline{L} \oplus \mathbf{Z} c, L^{\prime}=\underline{L^{\prime}} \oplus \mathbf{Z} c^{\prime}$.

In case (A) we set $I=\underline{I}$.

In case (B) we write $\underline{R}=\sqcup_{\mathfrak{e} \in \mathfrak{E}} \underline{R}_{\mathfrak{e}}, \underline{R}^{\prime}=\sqcup_{\mathfrak{e} \in \mathfrak{E}} \underline{R}_{\mathfrak{c}}^{\prime}$ where $\left(\underline{R}_{\mathfrak{e}}, \underline{R}_{\mathfrak{e}}^{\prime}\right)$ are irreducible root systems and $\mathfrak{E}$ is an indexing set; we have a corresponding partition $\underline{I}=\sqcup_{\mathfrak{e} \in \mathfrak{E}} \underline{I}_{\mathfrak{e}}$ where $\underline{I}_{\mathfrak{e}}$ indexes the simple roots/coroots in $\underline{R}_{\mathfrak{e}}, \underline{R}_{\mathfrak{e}}^{\prime}$. For each $\mathfrak{e} \in \mathfrak{E}$ let $\beta_{\mathfrak{e}} \in \underline{R}_{\mathfrak{e}}$ be such that $-\beta_{\mathfrak{e}}^{\prime}$ is the highest root of $\underline{R}_{\mathfrak{e}}^{\prime}$. We set $I=\underline{I} \sqcup \mathfrak{E}$. There is a unique function $\delta: \underline{R} \rightarrow \mathbf{Z}_{>0}$ such that $\delta_{\beta_{\mathrm{e}}}=1$ for any $\mathfrak{e} \in \mathfrak{E}$ and for any $h, \tilde{h}$ in $\underline{R}$ we have $\left\langle h, \tilde{h}^{\prime}\right\rangle \delta_{\tilde{h}}=\left\langle\tilde{h}, h^{\prime}\right\rangle \delta_{h}$. For any $h \in \underline{R}$ we have $\delta_{h} \in\{1,2,3\}$. For $\mathfrak{e} \in \mathfrak{E}$ we set $h_{\mathfrak{e}}=\beta_{\mathfrak{e}}+c \in L, h_{\mathfrak{e}}^{\prime}=\beta_{\mathfrak{e}}^{\prime} \in L^{\prime}$.

In both cases, for $i \in I$ we define a reflection $s_{i}: V \rightarrow V$ by $s_{i}(y)=$ $y-\left\langle y, h_{i}^{\prime}\right\rangle h_{i}$ and a reflection $s_{i}: V^{\prime} \rightarrow V^{\prime}$ by $s_{i}(x)=x-\left\langle h_{i}, x\right\rangle h_{i}^{\prime}$ (this is the contragredient of $s_{i}: V \rightarrow V$ ). Let $W$ (resp. $\underline{W}$ ) be the subgroup of $G L(V)$ generated by $\left\{s_{i} ; i \in I\right\}$ (resp. by $\left\{s_{i} ; i \in \underline{I}\right\}$ ); taking contragredients we identify $W$ (resp. $\underline{W}$ ) with the subgroup of $G L\left(V^{\prime}\right)$ generated by $\left\{s_{i} ; i \in I\right\}$ (resp. by $\left.\left\{s_{i} ; i \in \underline{I}\right\}\right)$. It is well known that $W(\operatorname{resp} \underline{W})$ is a Coxeter group on the set of generators $\left\{s_{i} ; i \in I\right\}$ (resp. $\left\{s_{i} ; i \in \underline{I}\right\}$ ). In any case $\underline{W}$ is a (finite) Weyl group. In case A we have $W=\underline{W}$; in case $\mathrm{B}, W$ is an affine Weyl group (said to be the affine Weyl group associated to $G$ ).

Let $w \mapsto|w|$ be the length function $W \rightarrow \mathbf{N}$; we set $S:=\left\{s_{i} ; i \in I\right\}=$ $\{w \in W ;|w|=1\}$. For $w \in W$ we have $w(L) \subset L, w\left(L^{\prime}\right) \subset L^{\prime}$. Hence the $W$-action on $V^{\prime}$ induces a $W$-action on $\bar{V}^{\prime}:=V^{\prime} / L^{\prime}$. For $y \in L, \lambda \in \bar{V}^{\prime}$ we define $(y, \lambda) \in \mathbf{Q} / \mathbf{Z}$ by $(y, \lambda)=\langle y, x\rangle$ where $x \in V^{\prime}$ is a representative of $\lambda$.

In case B we have $V^{\prime}=\sqcup_{\tilde{e} \in \mathbf{Q}} V_{\tilde{e}}^{\prime}$ where $V_{\tilde{e}}^{\prime}=\left\{x \in V^{\prime} ;\langle c, x\rangle=\tilde{e}\right\}$. The image of $V_{\tilde{e}}^{\prime}$ under $V^{\prime} \rightarrow \bar{V}^{\prime}=V^{\prime} / L^{\prime}$ depends only on the image $e$ of $\tilde{e}$ under the obvious map $\mathbf{Q} \rightarrow \mathbf{Q} / \mathbf{Z}$; we denote it by $\bar{V}_{e}^{\prime}$. We have $\bar{V}^{\prime}=\sqcup_{e \in \mathbf{Q} / \mathbf{Z}} \bar{V}_{e}^{\prime}$. In case A we sometimes write $\bar{V}_{0}^{\prime}$ instead of $\bar{V}^{\prime}$. We show:

(a) Any $W$-orbit on $\bar{V}^{\prime}$ is finite.

In case $\mathrm{A}$ this is trivial since $W$ is finite. Assume now that we are in case 
B. Let $\lambda \in \bar{V}^{\prime}$ and let $x \in V^{\prime}$ be a representative of $\lambda$. We can find an integer $q \geq 1$ such that $q x \in L^{\prime}$. Let $\mathcal{Q}$ be the subgroup of $V^{\prime}$ generated by $\left\{\delta_{h} h^{\prime} ; h \in \underline{R}\right\}$; this is the free abelian group with basis $\left\{\delta_{h_{i}} h_{i}^{\prime} ; i \in \underline{I}\right\}$. For $z \in \mathcal{Q}$ let $\theta_{z}: V^{\prime} \rightarrow V^{\prime}$ be the linear map $x \mapsto x+\langle c, x\rangle z$; its contragredient is the linear map $y \mapsto y-\langle y, z\rangle c, V \rightarrow V$. We have $\theta_{z} \in W$ (compare [5, 1.5]) and $z \mapsto \theta_{z}$ identifies $\mathcal{Q}$ with a normal subgroup $\mathcal{Q}^{\prime}$ of $W$ such that $W=\mathcal{Q}^{\prime} \underline{W}$ (semidirect product).

If $z \in \mathcal{Q}$ then $\theta_{q z}(x)-x=\langle c, q x\rangle z \in \mathcal{Q} \subset L^{\prime}$. Thus the stabilizer of $\lambda$ in $W$ contains $\mathcal{Q}^{\prime q}$. Thus the $W$-orbit of $\lambda$ contains at most $\sharp\left(W /\left(\mathcal{Q}^{\prime q}\right)\right)$ elements. Since $\sharp\left(W / \mathcal{Q}^{\prime}\right)<\infty$ and $\sharp\left(\mathcal{Q}^{\prime} / \mathcal{Q}^{\prime q}\right)<\infty$, we have $\sharp\left(W /\left(\mathcal{Q}^{\prime q}\right)\right)<$ $\infty$. This proves (a).

1.2. In case $\mathrm{A}$ we set $R=\underline{R}$. In case $\mathrm{B}$ we set

$$
R=\left\{\chi \in V ; \chi=w\left(h_{i}\right) \text { for some } i \in I \text { and some } w \in W\right\}
$$

we have $R=\left\{h+\delta_{h} m c ; h \in \underline{R}, m \in \mathbf{Z}\right\}$. In this case for $\chi \in R$ we set $\chi^{\prime}=h^{\prime} \in \underline{R}^{\prime}$ where $\chi=h+\delta_{h} m c$ with $h \in \underline{R}, m \in \mathbf{Z}$. The map $R \rightarrow \underline{R}^{\prime}$, $\chi \mapsto \chi^{\prime}$ extends the map $\underline{R} \rightarrow \underline{R}^{\prime}, h \mapsto h^{\prime}$ considered earlier and it is compatible with the notation $h_{\mathfrak{e}}^{\prime}$ considered earlier.

In both cases, for $\chi \in R$, the reflection $\sigma_{\chi}: V \rightarrow V, y \mapsto y-\left\langle y, \chi^{\prime}\right\rangle \chi$ and its contragredient $\sigma_{\chi}: V^{\prime} \rightarrow V^{\prime}, x \mapsto x-\langle\chi, x\rangle \chi^{\prime}$ are defined and belong to $W$. Let $V^{+}=\sum_{i \in I} \mathbf{Q}_{\geq 0} h_{i} \subset V$. We set $R^{+}=R \cap V^{+}, R^{-}=-R^{+}$.

Let $i_{1}, i_{2}, \ldots, i_{r}$ be a sequence in $I$ such that $w:=s_{i_{1}} s_{i_{2}} \ldots s_{i_{r}}$ satisfies $|w|=r$. A standard argument shows that

(a) $\left\{\chi \in R^{+} ; w(\chi) \in R^{-}\right\}$consists of exactly $r$ elements, namely $s_{i_{r}} s_{i_{r-1}} \ldots s_{i_{j+1}}\left(h_{i_{j}}\right)$ for $j=1, \ldots, r$.

For $\lambda \in \bar{V}^{\prime}$ we set $R_{\lambda}=\{\chi \in R ;(\chi, \lambda)=0 \in \mathbf{Q} / \mathbf{Z}\}, R_{\lambda}^{+}=R_{\lambda} \cap R^{+}$, $R_{\lambda}^{-}=R_{\lambda} \cap R^{-}$; let $W_{\lambda}^{\prime}=\{w \in W ; w(\lambda)=\lambda\}$, a subgroup of $W$. Let $W_{\lambda}$ be the subgroup of $W$ generated by $\left\{s_{\chi} ; \chi \in R_{\lambda}\right\}$. We have $W_{\lambda} \subset W_{\lambda}^{\prime}$. If $w^{\prime} \in W_{\lambda}^{\prime}$ and $\chi \in R_{\lambda}$, then $w^{\prime}(\chi) \in R_{\lambda}$; we have $w^{\prime} s_{\chi} w^{\prime-1}=s_{w^{\prime}(\chi)}$ hence $w^{\prime} W_{\lambda} w^{\prime-1}=W_{\lambda}$, so that $W_{\lambda}$ is a normal subgroup of $W_{\lambda}^{\prime}$.

In case A we set $\underline{R}_{\lambda}=R_{\lambda}$. In case B we set $\underline{R}_{\lambda}=\{h \in \underline{R} ; h+$ $m \delta_{h} c \in R_{\lambda}$ for some $\left.m \in \mathbf{Z}\right\}$; in the case where $\lambda \in \bar{V}_{0}^{\prime}$ we have $\underline{R}_{\lambda}=$ 
$\{h \in \underline{R} ;(h, \lambda)=0 \in \mathbf{Q} / \mathbf{Z}\}$. In both cases we set $\underline{R}_{\lambda}^{\prime}=\left\{h^{\prime} ; h \in \underline{R}_{\lambda}\right\} \subset \underline{R}^{\prime}$. We show that for $h, \tilde{h} \in \underline{R}_{\lambda}$ we have ${ }^{1} h:=s_{\tilde{h}}(h) \in \underline{R}_{\lambda}$.

Assume first that we are in case B. We have $\left(h+m \delta_{h} c, \lambda\right)=0,(\tilde{h}+$ $\left.\tilde{m} \delta_{\tilde{h}} c, \lambda\right)=0$ for some $m, \tilde{m}$ in $\mathbf{Z}$. We have ${ }^{1} h=h-\left\langle h, \tilde{h}^{\prime}\right\rangle \tilde{h} \in \underline{R}, \delta^{1} h=\delta_{h}$. Let ${ }^{1} m=m-\left\langle\tilde{h}, h^{\prime}\right\rangle \tilde{m} \in \mathbf{Z}$. We have

$$
\begin{aligned}
\left({ }^{1} h+{ }^{1} m \delta_{1_{h}} c, \lambda\right) & =\left(h-\left\langle h, \tilde{h}^{\prime}\right\rangle \tilde{h}+{ }^{1} m \delta_{h} c, \lambda\right) \\
& =-m \delta_{h}(c, \lambda)-\left\langle h, \tilde{h}^{\prime}\right\rangle(\tilde{h}, \lambda)+{ }^{1} m \delta_{h}(c, \lambda) \\
& =-m \delta_{h}(c, \lambda)+\left\langle h, \tilde{h}^{\prime}\right\rangle \tilde{m} \delta_{\tilde{h}}(c, \lambda)+{ }^{1} m \delta_{h}(c, \lambda) \\
& =-m \delta_{h}(c, \lambda)+\left\langle\tilde{h}, h^{\prime}\right\rangle \delta_{h}(c, \lambda)+{ }^{1} m \delta_{h}(c, \lambda)=0 .
\end{aligned}
$$

Thus $\left({ }^{1} h+{ }^{1} m \delta_{1} h c, \lambda\right)=0$ so that ${ }^{1} h \in \underline{R}_{\lambda}$. The proof in case A is the same (we formally set $m=\tilde{m}={ }^{1} m=0$ in the computation above.) We see that $\left(\underline{R}_{\lambda}, \underline{R}_{\lambda}\right)$ is a root system.

We show:

(b) For $\lambda \in \bar{V}^{\prime}, W_{\lambda}$ is a Coxeter group with length function $w \mapsto|w|_{\lambda}=$ $\sharp\left(\chi \in R_{\lambda}^{+} ; w(\chi) \in R_{\lambda}^{-}\right)$. Moreover, in case $A, W_{\lambda}$ is a Weyl group; in case $B, W_{\lambda}$ is an affine Weyl group. If $s \in W_{\lambda},|s|_{\lambda}=1$, then $s=s_{\chi}$ where $\chi$ is the unique element of $R_{\lambda}^{+}$such that $s(\chi) \in R_{\lambda}^{-}$.

In case A, $W_{\lambda}$ is the Weyl group of $\left(\underline{R}_{\lambda}, \underline{R}_{\lambda}^{\prime}\right)$ and (b) follows. We now assume that we are in case B. For any $\chi \in R_{\lambda}$ let $\mathcal{H}_{\chi}=\left\{x \in V_{1}^{\prime} ;\langle\chi, x\rangle=0\right\}$, a hyperplane in the affine space $V_{1}^{\prime}$. For $w \in W_{\lambda}, \chi \in R_{\lambda}$ we have $w\left(\mathcal{H}_{\chi}\right)=$ $\mathcal{H}_{w^{-1}(\chi)}$. For $x, x^{\prime}$ in $V_{1}^{\prime}-\cup_{\chi \in R_{\lambda}} \mathcal{H}_{\chi}$ we say that $x \sim x^{\prime}$ if $\langle\chi, x\rangle\left\langle\chi, x^{\prime}\right\rangle>0$ for any $\chi \in R_{\lambda}$; this is an equivalence relation on $V_{1}^{\prime}-\cup_{\chi \in R_{\lambda}} \mathcal{H}_{\chi}$; the equivalence classes are called $\lambda$-alcoves. There is a unique $\lambda$-alcove $C_{\lambda}$ that contains $\left\{x \in V_{1}^{\prime} ;\left\langle h_{i}, x\right\rangle>0 \forall i \in I\right\}$. Let $P_{\lambda}$ be the set of all $\chi \in R_{\lambda}^{+}$such that $\mathcal{H}_{\chi}$ is a wall of $C_{\lambda}$ (that is, the closure of $C_{\lambda}$ intersected with $\mathcal{H}_{\chi}$ is not contained in any affine subspace of $\mathcal{H}_{\chi}$ other than $\mathcal{H}_{\chi}$ ). We can apply the results in [1, Ch.V, §3], especially Theorem 1 ; we see that $W_{\lambda}$ is a Coxeter group on the generators $\left\{s_{\chi} ; \chi \in P_{\lambda}\right\}$ and with the length function as in (b). To prove the remaining statements of (b) we denote by $\underline{R}_{\lambda}^{u}(u \in \mathcal{U})$ the irreducible components of $\underline{R}_{\lambda}$. Note that if $u \neq u^{\prime}$ in $\mathcal{U}$, we have $\left\langle h, \tilde{h}^{\prime}\right\rangle=0$ for $h \in$ $\underline{R}_{\lambda}^{u}, \tilde{h} \in \underline{R}_{\lambda}^{u^{\prime}}$. For $u \in \mathcal{U}$ let $E_{u}$ be the affine subspace $c^{\prime}+\sum_{h \in \underline{R}_{\lambda}^{u}} \mathbf{Q} h^{\prime}$ of $V_{1}^{\prime}$; let $R_{\lambda}^{u}=\left\{h+m \delta_{h} c \in R_{\lambda} ; h \in \underline{R}_{\lambda}^{u}, m \in \mathbf{Z}\right\}$ and let $W_{\lambda}^{u}$ be the subgroup of $W_{\lambda}$ generated by $\left\{s_{\chi} ; \chi \in R_{\lambda}^{u}\right\}$. Now for $\chi \in R_{\lambda}^{u}$, the affine space $E_{u}$ is stable 
under $s_{\chi}: V^{\prime} \rightarrow V^{\prime}$; hence $E_{u}$ is stable under $W_{\lambda}^{u}$. We have $W_{\lambda}=\prod_{u \in \mathcal{U}} W_{\lambda}^{u}$. It is enough to show that for any $u \in \mathcal{U}, W_{\lambda}^{u}$ is an affine Weyl group. For any $\chi \in R_{\lambda}^{u}$ let $\mathcal{H}_{\chi, u}=\left\{x \in E_{u} ;\langle\chi, x\rangle=0\right\}=\mathcal{H}_{\chi} \cap E_{u}$, a hyperplane in $E_{u}$. For $x, x^{\prime}$ in $E_{u}-\cup_{\chi \in R_{\lambda}^{u}} \mathcal{H}_{\chi, u}$ we say that $x \sim x^{\prime}$ if $\langle\chi, x\rangle\left\langle\chi, x^{\prime}\right\rangle>0$ for any $\chi \in R_{\lambda, u}$; this is an equivalence relation on $E_{u}-\cup_{\chi \in R_{\lambda}^{u}} \mathcal{H}_{\chi, u}$; the equivalence classes are called the alcoves of $E_{u}$. Using again the results in [1, Ch.V, §3], we see that it is enough to show that some (or equivalently any) alcove in $E_{u}$ is bounded. Let $h^{j}, j=1 \ldots, t$ be the simple coroots in $\underline{R}_{\lambda}^{u}$ with respect to the set of positive coroots $\underline{R}_{\lambda}^{u} \cap V^{+}$. Let $h^{0}$ be a coroot in $\underline{R}_{\lambda}^{u}$ of the form $\sum_{j \in[1, t]} n_{j} h^{j}$ where $n_{j} \in \mathbf{Z}_{>0}$ for all $j$. For each $j \in[1, t]$ we can find $m_{j} \in \mathbf{Z}_{\geq 0}$ such that $\chi^{j}:=h^{j}+m_{j} \delta_{j} c \in R_{\lambda}^{u}$ (here $\delta_{j}=\delta_{h^{j}}$ ). We can find $m_{0} \in \mathbf{Z}_{>0}$ such that $\chi^{0}:=-h^{0}+m_{0} \delta_{0} c \in R_{\lambda}^{u}\left(\right.$ here $\left.\delta_{0}=\delta_{h^{0}}\right)$. Let

$$
\begin{aligned}
X & =\left\{x \in E_{u} ;\left\langle\chi^{j}, x\right\rangle>0 \forall j \in[0, t]\right\} \\
& =\left\{x \in E_{u} ;\left\langle h^{j}, x\right\rangle>-m_{j} \delta_{j} \forall j \in[1, t], \sum_{j \in[1, t]} n_{j}\left\langle h^{j}, x\right\rangle<m_{0} \delta_{0}\right\} .
\end{aligned}
$$

Note that $X$ contains at least one alcove and is bounded. (If $x \in E_{u}$, then $-m_{j} \delta_{j}<\left\langle h^{j}, x\right\rangle<m_{0} \delta_{0} / n_{j}$ for all $j \in[1, t]$.) It follows that some alcove in $E_{u}$ is bounded. This completes the proof of (b).

We show:

(c) Let $\lambda \in \bar{V}^{\prime}$. If $i \in I$ is such that $s_{i} \in W_{\lambda}$ then $h_{i} \in R_{\lambda}$.

We have $\left|s_{i}\right|_{\lambda}=\sharp\left(\chi \in R_{\lambda}^{+} ; s_{i}(\chi) \in R_{\lambda}^{-}\right) \leq \sharp\left(\chi \in R^{+} ; s_{i}(\chi) \in R-\right)=1$. Thus $\left|s_{i}\right|_{\lambda} \leq 1$. Since $s_{i}$ is an element of $W_{\lambda}$ other than 1 we have $\left|s_{i}\right|_{\lambda} \geq 1$ hence $\left|s_{i}\right|_{\lambda}=1$. By (b) we have $s_{i}=s_{\chi}$ for a unique $\chi \in R_{\lambda}^{+}$, hence $\chi=h_{i}$. This proves (c).

Let $\lambda \in \bar{V}^{\prime}$. Let $i_{1}, i_{2}, \ldots, i_{r}$ be a sequence in $I$ such that $w:=s_{i_{1}} s_{i_{2}} \ldots s_{i_{r}}$ $\in W_{\lambda}^{\prime},|w|=r$. Define $\mathcal{I}=\left\{j \in[1, r] ; s_{i_{r}} \ldots s_{i_{j+1}} s_{i_{j}} s_{i_{j+1}} \ldots s_{i_{r}} \in W_{\lambda}\right.$, $X=\left\{\chi \in R_{\lambda}^{+} ; w(\chi) \in R_{\lambda}^{-}\right\}$. We show:

(d) $\sharp(\mathcal{I})=\sharp(X)$.

Let $j \in \mathcal{I}$. Then $s_{i_{j}} \in W_{s_{i_{j+1}} \ldots s_{i_{r}}(\lambda)}$ hence by (c) we have $h_{i_{j}} \in$ $R_{s_{i_{j+1}} \ldots s_{i_{r}}(\lambda)}$ so that $s_{i_{r}} \ldots s_{i_{j+1}}\left(h_{i_{j}}\right) \in R_{\lambda}$. By (a), $s_{i_{r}} \ldots s_{i_{j+1}}\left(h_{i_{j}}\right)(j \in \mathcal{I})$ are distinct in $\left\{\chi \in R^{+} ; w(\chi) \in R^{-}\right\}$. Thus, $j \mapsto s_{i_{r}} \ldots s_{i_{j+1}}\left(h_{i_{j}}\right)$ is an injective $\operatorname{map} \phi: \mathcal{I} \rightarrow X$. 
Now let $\chi \in X$. From (a) we see that for some $j \in[1, r]$ we have $\chi=s_{i_{r}} \ldots s_{i_{j+1}}\left(h_{i_{j}}\right)$. Since $\chi \in R_{\lambda}$ we have

$$
h_{i_{j}} \in R_{s_{i_{j+1}} \ldots s_{i_{r}}(\lambda)}
$$

so that

$$
s_{i_{j}} \in W_{s_{i_{j+1}} \ldots s_{i_{r}}(\lambda)}
$$

and therefore $j \in \mathcal{I}$. We see that $\phi$ is surjective hence a bijection. This proves (d).

We show:

(e) Any right coset $w W_{\lambda} \subset W(w \in W)$ contains a unique element $w_{1}$ such that $w_{1}\left(R_{\lambda}^{+}\right) \subset R^{+}$. We have $\left|w_{1}\right|<\left|w_{1} z\right|$ for any $z \in W_{\lambda}-\{1\}$. We write $w_{1}=\min \left(w W_{\lambda}\right)$.

In case $\mathrm{A}$ this is proved in [3, 1.9]. The following proof applies in both cases A,B. We can find $w_{1} \in w W_{\lambda}$ such that $\left|w_{1}\right| \leq\left|w_{1} z\right|$ for any $z \in W_{\lambda}$. For any $\chi \in R_{\lambda}^{+}$we have $\left|w_{1} s_{\chi}\right| \neq\left|w_{1}\right|$ hence $\left|w_{1}\right|<\left|w_{1} s_{\chi}\right|$. Let $i_{1}, i_{2}, \ldots, i_{r}$ be a sequence in $I$ such that $s_{\chi} w_{1}^{-1}=s_{i_{1}} s_{i_{2}} \ldots s_{i_{r}} .\left|s_{\chi} w_{1}^{-1}\right|=r$. Since $\left|s_{\chi}\left(s_{c} w_{1}^{-1}\right)\right|<\left|s_{\chi} w_{1}^{-1}\right|$, we see from [6, 2.2] that, for some $f \in[1, r]$ we have $s_{\chi}=s_{i_{1}} s_{i_{2}} \ldots s_{i_{f}} \ldots s_{i_{2}} s_{i_{1}}$, hence $\chi=s_{i_{1}} s_{i_{2}} \ldots s_{i_{f-1}}\left(h_{i_{f}}\right)$. Applying (a) with $w$ replaced by $w_{1} s_{\chi}=s_{i_{r}} s_{i_{r-1}} \ldots s_{i_{1}}$, we see that $w_{1} s_{\chi}(\chi) \in R^{-}$that is $w_{1}(\chi) \in R^{+}$. We have thus shown that $w_{1}\left(R_{\lambda}^{+}\right) \subset R^{+}$. It follows that $w_{1}\left(R_{\lambda}^{-}\right) \subset R^{-}$. Now let $u \in W_{\lambda}-\{1\}$. We can find $\chi \in R_{\lambda}^{+}$such that $u(\chi) \in R_{\lambda}^{-}$. We then have $w_{1} u(\chi) \in R^{-}$. By the first part of the proof, $w_{1} u$ is not of minimal length in $w W_{\lambda}$. Thus $\left|w_{1}\right|<\left|w_{1} u\right|$. This proves (e).

Let $\mathfrak{o}$ be a $W$-orbit in $\bar{V}^{\prime}$. For $\lambda, \lambda^{\prime}$ in o let

$$
\begin{aligned}
{\left[\lambda^{\prime}, \lambda\right] } & =\left\{z \in W ; \lambda^{\prime}=z(\lambda), z=\min \left(z W_{\lambda}\right)\right\} \\
& =\left\{z \in W ; \lambda^{\prime}=z(\lambda), z\left(R_{\lambda}^{+}\right)=R_{\lambda^{\prime}}^{+}\right\}
\end{aligned}
$$

Clearly, $\left[\lambda, \lambda^{\prime}\right]=\left[\lambda^{\prime}, \lambda\right]^{-1}$ and for $\lambda^{\prime \prime} \in \mathfrak{o}$ we have $\left[\lambda^{\prime \prime}, \lambda^{\prime}\right]\left[\lambda^{\prime}, \lambda\right] \subset\left[\lambda^{\prime \prime}, \lambda\right]$. Thus,

(f) the group structure on $W$ makes $\Gamma:=\sqcup_{\left(\lambda^{\prime}, \lambda\right) \in \mathfrak{o}^{2}}\left[\lambda^{\prime}, \lambda\right]$ into a groupoid. 
In particular, for $\lambda \in \mathfrak{o}$,

$$
[\lambda, \lambda]=\left\{z \in W_{\lambda}^{\prime} ; z=\min \left(z W_{\lambda}\right)\right\}=\left\{z \in W_{\lambda}^{\prime} ; z\left(R_{\lambda}^{+}\right)=R_{\lambda}^{+}\right\}
$$

is a subgroup of $W_{\lambda}^{\prime}$. We show:

(g) For $\lambda \in \bar{V}^{\prime}$, the group $W_{\lambda}^{\prime}$ is the semidirect product of $[\lambda, \lambda]$ and $W_{\lambda}$ with $W_{\lambda}$ normal in $W_{\lambda}^{\prime}$.

By (e) we have $W_{\lambda}^{\prime}=[\lambda, \lambda] W_{\lambda}$ and $[\lambda, \lambda] \cap W_{\lambda}=\{1\}$. It remains to recall that $W_{\lambda}$ is normal in $W_{\lambda}^{\prime}$.

1.3. In this subsection we assume that we are in case B. We fix $e \in \mathbf{Q} / \mathbf{Z}$, $\lambda \in \bar{V}_{e}^{\prime}$ and a representative $x$ for $\lambda$ in $V^{\prime}$ such that $x \in V_{\tilde{e}}^{\prime}$ where $0 \leq \tilde{e}<1$. We write $\tilde{e}=p^{\prime} / q^{\prime}$ where $p^{\prime}, q^{\prime}$ are integers, $q^{\prime} \geq 1,0 \leq p^{\prime}<q^{\prime}$ and $p^{\prime}, q^{\prime}$ have no common prime divisor.

Assume first that $e=0$; then $\tilde{e}=0$ so that $\langle c, x\rangle=0$. It follows that

$$
R_{\lambda}=\left\{h+m \delta_{h} c ; h \in \underline{R}_{\lambda}, m \in \mathbf{Z}\right\}
$$

We write $\underline{R}_{\lambda}=\sqcup_{u \in \mathcal{U}} \underline{R}_{\lambda}^{u}$ as in the proof of $1.2(\mathrm{~b})$. Let $h^{u, 1}, \ldots, h^{u, t_{u}}$ be the set of simple coroots of $\underline{R}_{\lambda}^{u}$ with respect to the set of positive coroots $\underline{R}_{\lambda}^{u} \cap V^{+}$ and let $h^{u, 0}$ be the coroot in $\underline{R}_{\lambda}^{u}$ such that $\left(h^{u, 0}\right)^{\prime}$ is minus the highest root of $\left(\underline{R}_{\lambda}^{u}\right)^{\prime}$. For $j \in\left[0, t_{u}\right]$, let $\delta_{u, j}=\delta_{h^{u, j}}$. Note that $h^{u, j} \in R_{\lambda}^{+}$for $j \in\left[1, t_{u}\right]$ and $h^{u, 0}+\delta_{u, 0} c \in R_{\lambda}^{+}$. Let $\chi \in R_{\lambda}^{+}$. We show that

$$
\chi \in \sum_{u \in \mathcal{U}, j \in\left[1, t_{u}\right]} \mathbf{N} h^{u, j}+\sum_{u \in \mathcal{U}} \mathbf{N}\left(h^{u, 0}+\delta_{u, 0} c\right) .
$$

We have $\chi=h+m \delta_{h} c$ where either $h \in \underline{R}_{\lambda}^{u}, m \in \mathbf{Z}_{>0}, u \in \mathcal{U}$ or $h \in$ $\underline{R}_{\lambda}^{u} \cap V^{+}, m=0, u \in \mathcal{U}$. If $h \in \underline{R}_{\lambda}^{u} \cap V^{+}, m=0, u \in \mathcal{U}$, then clearly $\chi \in \sum_{j \in\left[1, t_{u}\right]} \mathbf{N} h^{u, j}$. If $h \in \underline{R}_{\lambda}^{u}, m \in \mathbf{Z}_{>0}, u \in \mathcal{U}$, then

$$
\begin{aligned}
\chi= & h+\delta_{h} m c=\left(h-\left(\delta_{h} / \delta_{u, 0}\right) h^{u, 0}\right)+\left(\delta_{h} / \delta_{u, 0}\right)(m-1)\left(-h^{u, 0}\right) \\
& +\left(\delta_{h} / \delta_{u, 0}\right) m\left(h^{u, 0}+\delta_{u, 0} c\right) .
\end{aligned}
$$

We now use $\delta_{h} / \delta_{u, 0} \in \mathbf{N}, h-\left(\delta_{h} / \delta_{u, 0}\right) h^{u, 0} \in \sum_{j \in\left[1, t_{u}\right]} \mathbf{N} h^{u, j}$ (a standard property of root systems) and (a) follows. Using (a) and the proof of $1.2(\mathrm{~b})$ 
we see that $W_{\lambda}$ is a Coxeter group on the generators

$$
\left.\left.s_{h^{u, j}} \text { with } u \in \mathcal{U}, j \in\left[1, t_{u}\right], \quad s_{h^{u, 0}+\delta_{u, 0} c} \text { with } u \in \mathcal{U}\right\}\right) .
$$

Next we assume that $e \neq 0$ (hence $0<p^{\prime}<q^{\prime}$ ) and $\underline{I}=\{1\}, I=$ $\underline{I} \sqcup\left\{i_{0}\right\}$, so that $\underline{R}=\left\{h_{1},-h_{1}\right\}, h_{i_{0}}=-h_{1}+c, h_{i_{0}}^{\prime}=-h_{1}^{\prime}$. We have $x=(p / q) h_{1}^{\prime} / 2+\left(p^{\prime} / q^{\prime}\right) c^{\prime}$, where $p, q$ are integers, $q \geq 1$ and $p, q$ have no common prime divisor. We have

$$
\begin{aligned}
R_{\lambda}^{+}= & \left\{h_{1}+m c ; m \in \mathbf{Z}_{\geq 0},(p / q)+\left(p^{\prime} / q^{\prime}\right) m \in \mathbf{Z}\right\} \\
& \sqcup\left\{-h_{1}+m c ; m \in \mathbf{Z}_{>0},-(p / q)+\left(p^{\prime} / q^{\prime}\right) m \in \mathbf{Z}\right\} .
\end{aligned}
$$

If $q^{\prime} \notin q \mathbf{Z}$ (so that $p \neq 0$ ), then the equation $\pm(p / q)+\left(p^{\prime} / q^{\prime}\right) m \in \mathbf{Z}$ has no integer solution $m$; hence in this case we have $R_{\lambda}=\emptyset$. We now assume that $q^{\prime} \in q \mathbf{Z}$. We have $\left\{m \in \mathbf{Z} ;(p / q)+\left(p^{\prime} / q^{\prime}\right) m \in \mathbf{Z}\right\}=m_{1}+q^{\prime} \mathbf{Z},\{m \in$ $\left.\mathbf{Z} ;-(p / q)+\left(p^{\prime} / q^{\prime}\right) m \in \mathbf{Z}\right\}=m_{2}+q^{\prime} \mathbf{Z}$, where $m_{1}, m_{2}$ are well defined integers in $\left[1, q^{\prime}-1\right]$ such that $p^{\prime}\left(m_{1}+m_{2}\right) \in q^{\prime} \mathbf{Z}$; thus we have $m_{2}=q^{\prime}-m_{1}$ and

$$
\begin{aligned}
R_{\lambda}^{+}= & \left\{h_{1}+m c ; m=m_{1}+q^{\prime} u, u \in \mathbf{Z}_{\geq 0}\right\} \\
& \sqcup\left\{-h_{1}+m c ; m=q^{\prime}-m_{1}+q^{\prime} u, u \in \mathbf{Z}_{\geq 0}\right\} .
\end{aligned}
$$

In particular, we have $h_{1}+m_{1} c \in R_{\lambda}^{+},-h_{1}+\left(q^{\prime}-m_{1}\right) c \in R_{\lambda}^{+}$. For $u \in \mathbf{Z}_{\geq 0}$ we have

$$
\begin{aligned}
h_{1}+\left(m_{1}+q^{\prime} u\right) c & =(u+1)\left(h_{1}+m_{1} c\right)+u\left(-h_{1}+\left(q^{\prime}-m_{1}\right) c\right), \\
-h_{1}+\left(q^{\prime}-m_{1}+q^{\prime} u\right) c & =u\left(h_{1}+m_{1} c\right)+(u+1)\left(-h_{1}+\left(q^{\prime}-m_{1}\right) c\right) .
\end{aligned}
$$

Thus $R_{\lambda}^{+} \subset \mathbf{N}\left(h_{1}+m_{1} c\right)+\mathbf{N}\left(-h_{1}+\left(q^{\prime}-m_{1}\right) c\right)$. Using this and the proof of $1.2(\mathrm{~b})$ we see that $W_{\lambda}$ is a Coxeter group (an infinite dihedral group) on the generators $s_{h_{1}+m_{1} c}, s_{-h_{1}+\left(q^{\prime}-m_{1}\right) c}$ (if $\left.q^{\prime} \in q \mathbf{Z}\right)$ and $W_{\lambda}=\{1\}$ if $q^{\prime} \notin q \mathbf{Z}$.

1.4. Until the end of 1.10 we fix a $W$-orbit $\mathfrak{o}$ in $\bar{V}^{\prime}$.

Let $H_{\mathfrak{o}}$ be the $\mathcal{A}$-algebra with 1 defined by the generators $T_{w}(w \in W)$ and $1_{\lambda}(\lambda \in \mathfrak{o})$ and relations:

$$
1_{\lambda} 1_{\lambda}=1_{\lambda} \text { for } \lambda \in \bar{V}^{\prime}, \quad 1_{\lambda} 1_{\lambda^{\prime}}=0 \text { for } \lambda \neq \lambda^{\prime} \text { in } \bar{V}^{\prime} \text {, }
$$




$$
\begin{gathered}
T_{w} T_{w^{\prime}}=T_{w w^{\prime}} \text { for } w, w^{\prime} \in W \text { such that }\left|w w^{\prime}\right|=|w|+\left|w^{\prime}\right|, \\
T_{w} 1_{\lambda}=1_{w(\lambda)} T_{w} \text { for } w \in W, \lambda \in \bar{V}^{\prime} \\
T_{s}^{2}=v^{2} T_{1}+\left(v^{2}-1\right) \sum_{\lambda \in \mathfrak{o} ; s \in W_{\lambda}} T_{s} 1_{\lambda} \text { for } s \in S \\
T_{1}=\sum_{\lambda \in \mathfrak{o}} 1_{\lambda} .
\end{gathered}
$$

Note that $T_{1}=\sum_{\lambda \in \mathfrak{o}} 1_{\lambda}$ is the unit element of $H_{\mathfrak{o}}$. It will be convenient to write $\tilde{T}_{w}=v^{-|w|} T_{w}$ for any $w \in W$. As in [7, 31.2], we see that

(a) $\left\{\tilde{T}_{w} 1_{\lambda} ; w \in W, \lambda \in \mathfrak{o}\right\}$ is an $\mathcal{A}$-basis of $H_{\mathfrak{o}}$.

From the definitions we have:

(b) if $s \in S, y \in W, \lambda \in \mathfrak{o}$ and $s \notin W_{y(\lambda)}$, then $\tilde{T}_{s} \tilde{T}_{y} 1_{\lambda}=\tilde{T}_{s y} 1_{\lambda}$.

We show:

(c) For $\lambda \in \mathfrak{o}, z \in[\lambda, \lambda], w \in W_{\lambda}^{\prime}$ we have $\tilde{T}_{z} \tilde{T}_{w} 1_{\lambda}=\tilde{T}_{z w} 1_{\lambda}, 1_{\lambda} \tilde{T}_{w^{-1}} \tilde{T}_{z^{-1}}=$ $1_{l} \tilde{T}_{w^{-1} z^{-1}}$.

The proof is an extension of the proof of [8,34.7(b)]. The use of the algebra antiautomorphism $\tilde{T}_{x} 1_{\lambda} \mapsto 1_{\lambda} \tilde{T}_{x^{-1}}$ shows that each of the two equalities in (c) implies the other equality. Let $i_{1}, i_{2}, \ldots, i_{r}$ be a sequence in $I$ such that $z^{-1}=s_{i_{1}} s_{i_{2}} \ldots s_{i_{r}},|z|=r$. Applying $1.2(\mathrm{~d})$ with $w$ replaced by $z^{-1}$ and noting that $X$ in $1.2(\mathrm{~d})$ is empty in this case, we see that $s_{i_{r}} \ldots s_{i_{j+1}} s_{i_{j}} s_{i_{j+1}} \ldots s_{i_{r}} \notin W_{\lambda}$ for any $j \in[1, r]$. From the relations of $H_{\mathfrak{o}}$ we have

$$
\tilde{T}_{w^{-1} s_{i_{1}} s_{i_{2}} \ldots s_{i_{j-1}}} \tilde{T}_{s_{i_{j}}} 1_{s_{i_{j+1}} \ldots s_{i_{r}} \lambda}=\tilde{T}_{w^{-1} s_{i_{1}} s_{i_{2}} \ldots s_{i_{j-1}} s_{i_{j}}} 1_{s_{i_{j+1}} \ldots s_{i_{r}} \lambda}
$$

for all $j \in[1, r]$. Using these identities we see that

$$
\tilde{T}_{w^{-1}} \tilde{T}_{z^{-1}} 1_{\lambda}=\tilde{T}_{w^{-1}} \tilde{T}_{s_{i_{1}}} \tilde{T}_{s_{i_{2}}} \ldots \tilde{T}_{s_{i_{r}}} 1_{\lambda}=\tilde{T}_{w^{-1} s_{i_{1}} s_{i_{2}} \ldots s_{i_{r}}} 1_{\lambda},
$$

and (c) follows.

The following result is a generalization of (c): 
(d) For $\left(\lambda^{\prime}, \lambda\right) \in \mathfrak{o}^{2}, z \in\left[\lambda^{\prime}, \lambda\right], w \in W_{\lambda}^{\prime}$ we have $\tilde{T}_{z} \tilde{T}_{w} 1_{\lambda}=\tilde{T}_{z w} 1_{\lambda}$, $1_{\lambda} \tilde{T}_{w^{-1}} \tilde{T}_{z^{-1}}=1_{l} \tilde{T}_{w^{-1} z^{-1}}$

The use of the algebra antiautomorphism $\tilde{T}_{x} 1_{\lambda} \mapsto 1_{\lambda} \tilde{T}_{x^{-1}}$ shows that each of the two equalities in (d) implies the other equality. We can find $r \geq 0$ and $i_{1}, i_{2}, \ldots, i_{r}$ in $I$ such that, setting $\lambda_{0}=\lambda, \lambda_{1}=s_{i_{1}}(\lambda), \lambda_{2}=$ $s_{i_{2}} s_{i_{1}}(\lambda), \lambda_{r}=s_{i_{r}} \ldots s_{i_{2}} s_{i_{1}}(\lambda)$, we have $\lambda_{0} \neq \lambda_{1} \neq \lambda_{2} \neq \cdots \neq \lambda_{r}=\lambda^{\prime}$. We have $s_{i_{1}} \in\left[\lambda_{1}, \lambda_{0}\right], s_{i_{2}} \in\left[\lambda_{2}, \lambda_{1}\right], \ldots, s_{i_{r}} \in\left[\lambda_{r}, \lambda_{r-1}\right]$, hence $s_{i_{r}} \ldots s_{i_{2}} s_{i_{1}} \in$ $\left[\lambda_{r}, \lambda_{0}\right]=\left[\lambda^{\prime}, \lambda\right]$. We define $\tilde{z} \in W$ by $z=s_{i_{r}} \ldots s_{i_{2}} s_{i_{1}} \tilde{z}$. Then $\tilde{z} \in[\lambda, \lambda]$. For $j \in[1, r]$ we have $s_{i_{j}} \notin W_{s_{i_{j-1}} \ldots s_{i_{1}}} \tilde{z}(\lambda)$ (since $\lambda_{i} \neq \lambda_{i-1}$ ) hence, using (b) twice, we have

$$
\begin{aligned}
& \tilde{T}_{s_{i_{j}}} \tilde{T}_{s_{i_{j-1}} \ldots s_{i_{1}}} \tilde{z} 1_{\lambda}=\tilde{T}_{s_{i_{j}} s_{i_{j-1} \ldots s_{i_{1}}}} 1_{\lambda}, \\
& \tilde{T}_{s_{i_{j}}} \tilde{T}_{s_{i_{j-1} \ldots s_{1}} \tilde{z} w} 1_{\lambda}=\tilde{T}_{s_{i_{j}} s_{i_{j-1} \ldots s_{i_{1}}} \tilde{z} w} 1_{\lambda} .
\end{aligned}
$$

Applying this repeatedly, we deduce

$$
\begin{gathered}
\tilde{T}_{s_{i_{r}} \ldots s_{i_{2}} s_{i_{1}}} \tilde{z} 1_{\lambda}=\tilde{T}_{s_{i_{r}}} \ldots \tilde{T}_{s_{i_{2}}} \tilde{T}_{s_{i_{1}}} \tilde{T}_{\tilde{z}} 1_{\lambda}, \\
\tilde{T}_{s_{i_{r} \ldots s_{i_{2}} s_{i_{1}}} \tilde{z} w} 1_{\lambda}=\tilde{T}_{s_{i_{r}}} \ldots \tilde{T}_{s_{i_{2}}} \tilde{T}_{s_{i_{1}}} \tilde{T}_{\tilde{z} w} 1_{\lambda} .
\end{gathered}
$$

By (c) we have $1_{\lambda} \tilde{T}_{w^{-1}} \tilde{T}_{\tilde{z}^{-1}}=1_{\lambda} \tilde{T}_{w^{-1} \tilde{z}^{-1}}$ and $\tilde{T}_{\tilde{z} w} 1_{\lambda}=\tilde{T}_{\tilde{z}} \tilde{T}_{w} 1_{\lambda}$. We deduce

$$
\begin{aligned}
\tilde{T}_{z w} 1_{\lambda}=\tilde{T}_{s_{i_{r}} \ldots s_{i_{2}} s_{i_{1}} \tilde{z} w} 1_{\lambda} & =\tilde{T}_{s_{i_{r}}} \ldots \tilde{T}_{s_{i_{2}}} \tilde{T}_{s_{i_{1}}} \tilde{T}_{\tilde{z} w} 1_{\lambda} \\
& =\tilde{T}_{s_{i_{r}}} \ldots \tilde{T}_{s_{i_{2}}} \tilde{T}_{s_{i_{1}}} \tilde{T}_{\tilde{z}} \tilde{T}_{w} 1_{\lambda}=\tilde{T}_{s_{i_{r}}} \ldots \tilde{T}_{s_{i_{2}}} \tilde{T}_{s_{i_{1}}} \tilde{T}_{\tilde{z}} 1_{\lambda} \tilde{T}_{w} \\
& =\tilde{T}_{s_{i_{r}} \ldots s_{i_{2}} s_{i_{1}}} \tilde{z}_{\lambda} \tilde{T}_{w}=\tilde{T}_{z} \tilde{T}_{w} 1_{\lambda} .
\end{aligned}
$$

This proves the first equality in (d); the second equality follows also.

We show:

(e) For $\lambda, \lambda^{\prime}, \lambda^{\prime \prime}$ in $\mathfrak{o}, z \in\left[\lambda^{\prime}, \lambda\right], z^{\prime} \in\left[\lambda, \lambda^{\prime \prime}\right]$ we have $\tilde{T}_{z} \tilde{T}_{z^{\prime}} 1_{\lambda^{\prime \prime}}=\tilde{T}_{z z^{\prime}} 1_{\lambda^{\prime \prime}}$.

As in the proof of (d) we have $z=s_{i_{r}} \ldots s_{i_{2}} s_{i_{1}} \tilde{z}$,

$\tilde{T}_{z} 1_{\lambda}=\tilde{T}_{s_{i_{r}}} \ldots \tilde{T}_{s_{i_{2}}} \tilde{T}_{s_{i_{1}}} \tilde{T}_{\tilde{z}} 1_{\lambda}$, where $i_{1}, \ldots i_{r} \in I, \lambda=\lambda_{0} \neq \lambda_{1} \neq \lambda_{2} \neq \cdots \neq$ $\lambda_{r}=\lambda^{\prime}$ are in $\bar{V}^{\prime}, \lambda_{0}=\lambda, \lambda_{1}=s_{i_{1}}(\lambda), \lambda_{2}=s_{i_{2}} s_{i_{1}}(\lambda), \lambda_{r}=s_{i_{r}} \ldots s_{i_{2}} s_{i_{1}}(\lambda)$, $\tilde{z} \in[\lambda, \lambda]$. Hence it is enough to show that

$$
\tilde{T}_{s_{i_{r}}} \ldots \tilde{T}_{s_{i_{2}}} \tilde{T}_{s_{i_{1}}} \tilde{T}_{\tilde{z}} \tilde{T}_{z^{\prime}} 1_{\lambda^{\prime \prime}}=\tilde{T}_{s_{i_{r}} \ldots s_{i_{2}} s_{i_{1}}} \tilde{z} z^{\prime} 1_{\lambda^{\prime \prime}}
$$


It is enough to show that for any $j \in[0, r]$ we have

$$
\tilde{T}_{s_{i_{j}}} \ldots \tilde{T}_{s_{i_{2}}} \tilde{T}_{s_{i_{1}}} \tilde{T}_{\tilde{z}} \tilde{T}_{z^{\prime}} 1_{\lambda^{\prime \prime}}=\tilde{T}_{s_{i_{j}} \ldots s_{i_{2}} s_{i_{1}}} \tilde{z} z^{\prime} 1_{\lambda^{\prime \prime}}
$$

We argue by induction on $j$. For $j=0$ we must show that $\tilde{T}_{\tilde{z}} \tilde{T}_{z^{\prime}} 1_{\lambda^{\prime \prime}}=$ $\tilde{T}_{\tilde{z} z^{\prime}} 1_{\lambda^{\prime \prime}} ;$ this follows from (d). Now assume that $j \geq 1$. Using the induction hypothesis we see that it is enough to show that

$$
\tilde{T}_{s_{j}} \tilde{T}_{w_{j}} 1_{\lambda^{\prime \prime}}=\tilde{T}_{s_{i_{j}} w_{j}} 1_{\lambda^{\prime \prime}}
$$

where $w_{j}=s_{i_{j-1}} \ldots s_{i_{2}} s_{i_{1}} \tilde{z} z^{\prime}$. Using (b) we see that it is enough to show that $s_{i_{j}} \notin W_{s_{i_{j-1}} \ldots s_{i_{2}} s_{i_{1}}} \tilde{z z^{\prime}\left(\lambda^{\prime \prime}\right)}$, or that $s_{i_{j}} \notin W_{\lambda_{j}}$. We have $s_{i_{j}}\left(\lambda_{j}\right)=\lambda_{j-1} \neq \lambda_{j}$ so that $s_{i_{j}} \notin W_{\lambda_{j}}$ as desired. This proves (e).

We show:

(f) Let $\lambda \in \mathfrak{o}, w \in W, \sigma \in W_{\lambda}$. Assume that $|\sigma|_{\lambda}=1$. We have $\tilde{T}_{w} \tilde{T}_{\sigma} 1_{\lambda}=$ $\tilde{T}_{w \sigma} 1_{\lambda}+\delta\left(v-v^{-1}\right) \tilde{T}_{w} 1_{\lambda}$ where $\delta \in\{0,1\}$. Moreover, if $w \in W_{\lambda}$ then $\delta=0$ if $|w \sigma|_{\lambda}>|w|_{\lambda}$ and $\delta=1$ if $|w \sigma|_{\lambda}<|w|_{\lambda}$.

In case A this follows from [8, 34.7(a)]. The following proof applies in both cases A,B. We have $\sigma=s_{i_{1}} s_{i_{2}} \ldots s_{i_{r}}$ for some sequence $i_{1}, i_{2}, \ldots, i_{r}$ in $I$ such that $r=|\sigma|$. From $1.2(\mathrm{~d})$ we see that there is a unique $j \in[1, r]$ such that

$$
s_{i_{r}} \ldots s_{i_{j+1}} s_{i_{j}} s_{i_{j+1}} \ldots s_{i_{r}} \in W_{\lambda}
$$

thus for $j^{\prime} \in[1, r]-\{j\}$ we have

$$
s_{i_{r}} \ldots s_{i_{j^{\prime}+1}} s_{i_{j^{\prime}}} s_{i_{j^{\prime}+1}} \ldots s_{i_{r}} \notin W_{\lambda}
$$

moreover from the proof of $1.2(\mathrm{~d})$ we see that $s_{i_{r}} \ldots s_{i_{j+1}}\left(h_{i_{j}}\right)$ is the unique element $\chi \in R_{\lambda}^{+}$such that $\sigma(\chi) \in R_{\lambda}^{-}$. It follows that the reflection in $W$ defined by $s_{i_{r}} \ldots s_{i_{j+1}}\left(h_{i_{j}}\right)$ coincides with the reflection defined by $\chi$; thus we have

$$
\sigma=s_{i_{r}} \ldots s_{i_{j+1}} s_{i_{j}} s_{i_{j+1}} \ldots s_{i_{r}} .
$$

It follows that

$$
s_{i_{1}} s_{i_{2}} \ldots s_{i_{j-1}} s_{i_{j+1}} \ldots s_{i_{r}}=1 \text {. }
$$


From the relations of $H_{\mathfrak{o}}$ we have

$$
\begin{aligned}
& \tilde{T}_{w s_{i_{1}} s_{i_{2}} \ldots s_{i_{j-1}}} \tilde{T}_{s_{i_{j}}} 1_{s_{i_{j+1}} \ldots s_{i_{r}}(\lambda)} \\
& =\tilde{T}_{w s_{i_{1}} s_{i_{2}} \ldots s_{i_{j}}} 1_{s_{i_{j+1}} \ldots s_{i_{r}}(\lambda)}+\delta^{\prime}\left(v-v^{-1}\right) \tilde{T}_{w s_{i_{1}} s_{i_{2}} \ldots s_{i_{j-1}}} 1_{s_{i_{j+1}} \ldots s_{i_{r}}}(\lambda)
\end{aligned}
$$

where $\delta^{\prime}=0$ if $\left|w s_{i_{1}} s_{i_{2}} \ldots s_{i_{j}}\right|>\left|w s_{i_{1}} s_{i_{2}} \ldots s_{i_{j-1}}\right|$ and $\delta^{\prime}=1$ otherwise,

$$
\tilde{T}_{w s_{i_{1}} s_{i_{2}} \ldots s_{i_{j^{\prime}-1}}} \tilde{T}_{s_{j_{j^{\prime}}}} 1_{s_{i_{j^{\prime}+1}} \ldots s_{i_{r}}(\lambda)}=\tilde{T}_{w s_{i_{1}} s_{i_{2}} \ldots s_{i_{j^{\prime}}}} 1_{s_{i_{j^{\prime}+1}} \ldots s_{i_{r}}(\lambda)}
$$

if $j^{\prime} \in[1, r]-\{j\}$,

$$
\begin{aligned}
& \tilde{T}_{w s_{i_{1}} s_{i_{2}} \ldots s_{i_{j-1}} s_{i_{j+1}} \ldots s_{i_{j^{\prime}-1}}} \tilde{T}_{s_{i_{j^{\prime}}}} 1_{s_{i_{j^{\prime}+1}} \ldots s_{i_{r}}(\lambda)} \\
& =\tilde{T}_{w s_{i_{1}} s_{i_{2}} \ldots s_{i_{j-1}}} s_{i_{j+1} \ldots s_{j^{\prime}}} 1_{s_{i_{j^{\prime}+1}} \ldots s_{i_{r}}(\lambda)}
\end{aligned}
$$

if $j^{\prime} \in[j+1, r]$. From these identities we see that

$$
\begin{aligned}
\tilde{T}_{w} \tilde{T}_{\sigma} 1_{\lambda}= & \tilde{T}_{w} \tilde{T}_{s_{i_{1}}} \tilde{T}_{s_{i_{2}}} \ldots \tilde{T}_{s_{i_{r}}} 1_{\lambda} \\
= & \tilde{T}_{w s_{i_{1}} s_{i_{2}} \ldots s_{i_{j-1}}} \tilde{T}_{s_{i_{j}}} \tilde{T}_{s_{i_{j+1}}} \ldots \tilde{T}_{s_{i_{r}}} 1_{\lambda} \\
= & \tilde{T}_{w s_{i_{1}} s_{i_{2}} \ldots s_{i_{j-1}}} s_{i_{j}} \tilde{T}_{s_{i_{j+1}}} \ldots \tilde{T}_{s_{i_{r}}} 1_{\lambda} \\
& +\delta^{\prime}\left(v-v^{-1}\right) \tilde{T}_{w s_{i_{1}} s_{i_{2}} \ldots s_{i_{j-1}}} \tilde{T}_{s_{i_{j+1}}} \ldots \tilde{T}_{s_{i_{r}}} 1_{\lambda} \\
= & \tilde{T}_{w s_{i_{1}} s_{i_{2}} \ldots s_{i_{j}} s_{i_{j+1}} \ldots s_{i_{r}}} 1_{\lambda} \\
& +\delta^{\prime}\left(v-v^{-1}\right) \tilde{T}_{w s_{i_{1}} s_{i_{2}} \ldots s_{i_{j-1}} s_{i_{j+1}} \ldots s_{i_{r}}} 1_{\lambda} \\
= & \tilde{T}_{w \sigma} 1_{\lambda}+\delta^{\prime}\left(v-v^{-1}\right) \tilde{T}_{w} 1_{\lambda} .
\end{aligned}
$$

Assume now that $w \in W_{\lambda}$. We show that $\delta=\delta^{\prime}$. The condition that $\delta=0$ is equivalent to the condition that $w(\chi) \in R_{\lambda}^{+}$. The condition that $\delta^{\prime}=0$ is equivalent to the condition that $w s_{i_{1}} s_{i_{2}} \ldots s_{i_{j-1}}\left(h_{i_{j}}\right) \in R^{+}$. It remains to note that $\chi=s_{i_{1}} s_{i_{2}} \ldots s_{i_{j-1}}\left(h_{i_{j}}\right)$. (This follows from $\chi=s_{i_{r}} \ldots s_{i_{j+1}}\left(h_{i_{j}}\right)$ since $s_{i_{r}} \ldots s_{i_{j+1}}=s_{i_{1}} s_{i_{2}} \ldots s_{i_{j-1}}$, or equivalently $s_{i_{1}} s_{i_{2}} \ldots s_{i_{j-1}} s_{i_{j+1}} \ldots s_{i_{r}}=$ 1.)

We show:

(g) Let $\lambda \in \mathfrak{o}, w \in W_{\lambda}, w^{\prime} \in W_{\lambda}$. Assume that $\left|w w^{\prime}\right|_{\lambda}=|w|_{\lambda}+\left|w^{\prime}\right|_{\lambda}$. We have $\tilde{T}_{w w^{\prime}} 1_{\lambda}=\left(\tilde{T}_{w} 1_{\lambda}\right)\left(\tilde{T}_{w^{\prime}} 1_{\lambda}\right)$. 
We argue by induction on $\left|w^{\prime}\right|_{\lambda}$. If $w^{\prime}=1$ the result is obvious. We now assume that $\left|w^{\prime}\right|_{\lambda} \geq 1$. We can write $w^{\prime}=w_{1}^{\prime} \sigma$ where $w_{1}^{\prime}, \sigma$ are in $W_{\lambda}$ and $\left|w_{1}^{\prime}\right|_{\lambda}=\left|w^{\prime}\right|_{\lambda}-1,|\sigma|_{\lambda}=1$. We have $\left|w w_{1}^{\prime}\right|_{\lambda}=|w|_{\lambda}+\left|w_{1}^{\prime}\right|_{\lambda}$. From (f) we have $\tilde{T}_{w^{\prime}} 1_{\lambda}=\left(\tilde{T}_{w_{1}^{\prime}} 1_{\lambda}\right)\left(\tilde{T}_{\sigma} 1_{\lambda}\right)$. Using this and the induction hypothesis we have

$$
\left(\tilde{T}_{w} 1_{\lambda}\right)\left(\tilde{T}_{w^{\prime}} 1_{\lambda}\right)=\left(\tilde{T}_{w} 1_{\lambda}\right)\left(\tilde{T}_{w_{1}^{\prime}} 1_{\lambda}\right)\left(\tilde{T}_{\sigma} 1_{\lambda}\right)=\left(\tilde{T}_{w w_{1}^{\prime}} 1_{\lambda}\right)\left(\tilde{T}_{\sigma} 1_{\lambda}\right) .
$$

It remains to use that $\left(\tilde{T}_{w w_{1}^{\prime}} 1_{\lambda}\right)\left(\tilde{T}_{\sigma} 1_{\lambda}\right)=\tilde{T}_{w w_{1}^{\prime} \sigma} 1_{\lambda}$ which follows again from (f).

The following result is a special case of $(f)$.

(h) Let $\lambda \in \mathfrak{o}, \sigma \in W_{\lambda}$ be such that $|\sigma|_{\lambda}=1$. We have $\left(\tilde{T}_{\sigma} 1_{\lambda}\right)^{2}=1_{\lambda}+(v-$ $\left.v^{-1}\right) \tilde{T}_{\sigma} 1_{\lambda}$.

1.5. For $\lambda \in \mathfrak{o}$ let $H_{\lambda}$ be the $\mathcal{A}$-submodule of $H_{\mathfrak{o}}$ with basis $\left\{\tilde{T}_{w} 1_{\lambda} ; w \in W_{\lambda}\right\}$. From $1.4(\mathrm{~g}),(\mathrm{h})$, we see that $H_{\lambda}$ is a subalgebra of $H_{\mathfrak{o}}$ (with a different unit, namely $\left.1_{\lambda}\right)$.

Let $\Xi=\left\{\left(\lambda^{\prime}, z, \lambda\right) ;\left(\lambda^{\prime}, \lambda\right) \in \mathfrak{o}^{2}, z \in\left[\lambda^{\prime}, \lambda\right]\right\}$. We show:

(a) Let $\left(\lambda^{\prime}, z, \lambda\right) \in \Xi$. The assignment $w \mapsto z w z^{-1}$ is a Coxeter group isomorphism $W_{\lambda} \stackrel{\sim}{\rightarrow} W_{\lambda^{\prime}}$. The assignment $\tilde{T}_{w} 1_{\lambda} \mapsto \tilde{T}_{z w z^{-1}} 1_{\lambda^{\prime}}\left(w \in W_{\lambda}\right)$ is an $\mathcal{A}$-algebra isomorphism $\iota_{z}: H_{\lambda} \stackrel{\sim}{\rightarrow} H_{\lambda^{\prime}}$.

Recall that $z\left(R_{\lambda}^{+}\right)=R_{\lambda^{\prime}}^{+}$. This shows that $w \mapsto z w z^{-1}$ defines an isomorphism $W_{\lambda} \rightarrow W_{\lambda^{\prime}}$. For any $w \in W_{\lambda}, \chi \mapsto z(\chi)$ defines a bijection $\left\{\chi_{1} \in R_{\lambda}^{+} ; w\left(\chi_{1}\right) \in R_{\lambda}^{-}\right\} \rightarrow\left\{\chi_{2} \in R_{\lambda^{\prime}}^{+} ; z w z^{-1}\left(\chi_{2}\right) \in R_{\lambda^{\prime}}^{-}\right\}$. It follows that $\left|z w z^{-1}\right|_{\lambda^{\prime}}=|w|_{\lambda}$ for any $w \in W_{\lambda}$. Thus, $w \mapsto z w z^{-1}$ is an isomorphism of Coxeter groups. We also see that $\tilde{T}_{w} 1 \lambda \mapsto \tilde{T}_{z w z^{-1}} 1_{\lambda^{\prime}}$ defines an isomorphism of $\mathcal{A}$-modules $\iota_{z}: H_{\lambda} \stackrel{\sim}{\rightarrow} H_{\lambda^{\prime}}$. To show that $\iota_{z}$ is compatible with multiplication it is enough to note that, by $1.4(\mathrm{~d}),(\mathrm{e})$, for $w \in W_{\lambda}$ we have $\tilde{T}_{z w z^{-1}} 1_{\lambda^{\prime}}=\tilde{T}_{z} \tilde{T}_{w} 1_{\lambda} \tilde{T}_{z}^{-1}$. This proves (a).

Let $\mathcal{R}$ be a commutative ring with 1 . Let $A l g_{\mathcal{R}}$ be the category of associative $\mathcal{R}$-algebras with 1 . Let $\Phi: \Gamma \rightarrow A l g_{\mathcal{R}}$ be a functor; here the groupoid $\Gamma$ (in $1.2(\mathrm{f})$ ) is viewed as a category in which all morphisms are isomorphisms. Now $\Phi$ consists of a collection $\{\Phi(\lambda) ; \lambda \in \mathfrak{o}\}$ of associative $\mathcal{R}$-algebras with 1 together with a collection of algebra isomorphisms $\iota_{z}: \Phi(\lambda) \stackrel{\sim}{\rightarrow} \Phi\left(\lambda^{\prime}\right)$ (one 
for each $\left.\left(\lambda^{\prime}, z, \lambda\right) \in \Xi\right)$ such that for any $\left(\lambda_{1}^{\prime}, z_{1}, \lambda_{1}\right),\left(\lambda_{2}^{\prime}, z_{2}, \lambda_{2}\right)$ in $\Xi$ with $\lambda_{2}^{\prime}=\lambda_{1}$, the compositions $\Phi\left(\lambda_{2}\right) \stackrel{\iota_{z_{2}}}{\rightarrow} \Phi\left(\lambda_{2}^{\prime}\right)=\Phi\left(\lambda_{1}\right) \stackrel{\iota_{z}}{\rightarrow} \Phi\left(\lambda_{1}^{\prime}\right), \Phi\left(\lambda_{2}\right) \stackrel{\iota_{z_{1} z_{2}}}{\longrightarrow} \Phi\left(\lambda_{1}^{\prime}\right)$ coincide.

Given $\Phi$ we define $\Phi^{\bullet} \in A g_{\mathcal{R}}$ as follows. As an $\mathcal{R}$-module we have $\Phi^{\bullet}=\oplus_{\left(\lambda^{\prime}, z, \lambda\right) \in \Xi} \Phi_{\lambda^{\prime}, z, \lambda}^{\bullet}$ where $\Phi_{\lambda^{\prime}, z, \lambda}^{\bullet}=\Phi(\lambda)$. For any $\left(\lambda_{1}^{\prime}, z_{1}, \lambda_{1}\right),\left(\lambda_{2}^{\prime}, z_{2}, \lambda_{2}\right)$ in $\Xi$ and any $\xi_{1} \in \Phi_{\lambda_{1}^{\prime}, z_{1}, \lambda_{1}}^{\bullet}=\Phi\left(\lambda_{1}\right), \xi_{2} \in \Phi_{\lambda_{2}^{\prime}, z_{2}, \lambda_{2}}^{\bullet}=\Phi\left(\lambda_{2}\right)$, we define the product $\xi_{1} \xi_{2} \in \Phi^{\bullet}$ as 0 if $\lambda_{2}^{\prime} \neq \lambda_{1}$ and as $\iota_{z_{2}}^{-1}\left(\xi_{1}\right) \xi_{2} \in \Phi_{\lambda_{1}^{\prime}, z_{1} z_{2}, \lambda_{2}}=\Phi\left(\lambda_{2}\right)$ (product in $\Phi\left(\lambda_{2}\right)$ ) if $\lambda_{2}^{\prime}=\lambda_{1}$. From the definitions we see that this product is associative with unit element such that its $\Phi_{\lambda^{\prime}, z, \lambda}^{\bullet}$-component is 0 unless $\lambda=\lambda^{\prime}, z=1$. in which case it is the unit element of $\Phi(\lambda)$.

1.6. Let $\mathcal{H}: \Gamma \rightarrow \operatorname{Alg}_{\mathcal{A}}$ be the functor defined by $\mathcal{H}(\lambda)=H_{\lambda}$ for $\lambda \in \mathfrak{o}$ and the isomorphisms $\iota_{z}: H_{\lambda} \stackrel{\sim}{\rightarrow} H_{\lambda^{\prime}}$ in 1.5(a) for $\left(\lambda^{\prime}, z, \lambda\right) \in \Xi$. Then the associative algebra $\mathcal{H}^{\bullet}=\oplus_{\left(\lambda^{\prime}, z, \lambda\right) \in \Xi} \mathcal{H}_{\lambda^{\prime}, z, \lambda}$ where $\mathcal{H}_{\lambda^{\prime}, z, \lambda}=H_{\lambda}$ is defined as in 1.5. We consider the $\mathcal{A}$-linear map $\theta: \mathcal{H}^{\bullet} \rightarrow H_{\mathfrak{o}}$ such that for any $\left(\lambda^{\prime}, z, \lambda\right) \in \Xi$ and any $\xi \in \mathcal{H}_{\lambda^{\prime}, z, \lambda}^{\bullet}=H_{\lambda}$ we have $\theta(\xi)=\tilde{T}_{z} \xi \in H_{\mathfrak{o}}$. From $1.2(\mathrm{e})$ we see that $\theta$ is an isomorphism of $\mathcal{A}$-modules. We show that it respects the algebra structures. For $\left(\lambda_{1}^{\prime}, z_{1}, \lambda_{1}\right),\left(\lambda_{2}^{\prime}, z_{2}, \lambda_{2}\right)$ in $\Xi$ and for $w_{1} \in W_{\lambda_{1}}, w_{2} \in W_{\lambda_{2}}$, we must show that $\tilde{T}_{z_{1}} \tilde{T}_{w_{1}} 1_{\lambda_{1}} \tilde{T}_{z_{2}} \tilde{T}_{w_{2}} 1_{\lambda_{2}}$ is zero if $\lambda_{1} \neq \lambda_{2}^{\prime}$, while if $\lambda_{1}=\lambda_{2}^{\prime}$, it is $\tilde{T}_{z_{1} z_{2}} \tilde{T}_{z_{2}^{-1} w_{1} z_{2}} \tilde{T}_{w_{2}} 1_{\lambda_{2}}$. The case where $\lambda_{1} \neq \lambda_{2}^{\prime}$ is immediate. The case where $\lambda_{1}=\lambda_{2}^{\prime}$ follows from 1.4(d),(e). We see that

(a) $\theta: \mathcal{H}^{\bullet} \rightarrow H_{\mathfrak{o}}$ is an isomorphism of $\mathcal{A}$-algebras.

Now, for $\lambda \in \mathfrak{o}$, let $\mathfrak{H}_{\lambda}$ be the Hecke algebra associated to the Coxeter group $W_{\lambda}$. Thus, $\mathfrak{H}_{\lambda}$ is the $\mathcal{A}$-module with basis $\left\{\mathfrak{T}_{w} ; w \in W_{\lambda}\right\}$ with the associative $\mathcal{A}$-algebra structure defined by the rules

$$
\begin{aligned}
& \mathfrak{T}_{w} \mathfrak{T}_{w^{\prime}}=\mathfrak{T}_{w w^{\prime}} \text { if } w, w^{\prime} \text { in } W_{\lambda} \text { satisfy }\left|w w^{\prime}\right|_{\lambda}=|w|_{\lambda}+\left|w^{\prime}\right|_{\lambda}, \\
& \mathfrak{T}_{\sigma}^{2}=1+\left(v-v^{-1}\right) \mathfrak{T}_{\sigma} \text { if } \sigma \in W_{\lambda} \text { satisfies }|\sigma|_{\lambda}=1 .
\end{aligned}
$$

Note that $\mathfrak{T}_{1}$ is the unit element of $\mathfrak{H}_{\lambda}$. We define an $\mathcal{A}$-linear map $\vartheta_{\lambda}$ : $\mathfrak{H}_{\lambda} \rightarrow H_{\lambda}$ by $\mathfrak{T}_{w} \mapsto \tilde{T}_{w} 1_{\lambda}$ for $w \in W_{\lambda}$. This is clearly an isomorphism of $\mathcal{A}$-modules; moreover, from $1.4(\mathrm{~g}),(\mathrm{h})$, we see that this is an algebra isomorphism.

Let $\mathcal{H}^{\prime}: \Gamma \rightarrow A l g_{\mathcal{A}}$ be the functor defined by $\mathcal{H}^{\prime}(\lambda)=\mathfrak{H}_{\lambda}$ for $\lambda \epsilon$ $\mathfrak{o}$ and the isomorphisms $\iota_{z}: \mathfrak{H}_{\lambda} \sim_{\rightarrow} \mathfrak{H}_{\lambda^{\prime}}, \mathfrak{T}_{w} \mapsto \mathfrak{T}_{z w z^{-1}}$ for $w \in W_{\lambda}$ (here 
$\left.\left(\lambda^{\prime}, z, \lambda\right) \in \Xi\right)$. Then the associative algebra $\mathcal{H}^{\prime \bullet}=\oplus_{\left(\lambda^{\prime}, z, \lambda\right) \in \Xi \mathcal{H}_{\lambda^{\prime}, z, \lambda}^{\prime}} \bullet$ where $\mathcal{H}_{\lambda^{\prime}, z, \lambda}^{\prime}=\mathfrak{H}_{\lambda}$ is defined as in 1.5 .

We consider the $\mathcal{A}$-linear map $\vartheta: \mathcal{H}^{\bullet} \rightarrow \mathcal{H}^{\bullet}$ such that for any $\left(\lambda^{\prime}, z, \lambda\right) \in$ $\Xi$ and any $\xi \in \mathcal{H}_{\lambda^{\prime}, z, \lambda}^{\prime}=\mathfrak{H}_{\lambda}$ we have $\vartheta(\xi)=\vartheta_{\lambda}(\xi) \in \mathcal{H}_{\lambda^{\prime}, z, \lambda}=H_{\lambda}$. Clearly, $\vartheta$ is an isomorphism of $\mathcal{A}$-modules. Moreover, $\vartheta$ is an isomorphism of algebras. (We use that for any $\left(\lambda^{\prime}, z, \lambda\right) \in \Xi$, the compositions $\mathfrak{H}_{\lambda} \stackrel{\vartheta_{\lambda}}{\rightarrow} H_{\lambda} \stackrel{\text { 年 }}{\rightarrow} H_{\lambda^{\prime}}$,

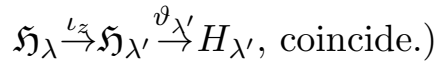

For $s \in S$ we have $\tilde{T}_{s}^{-1}=\tilde{T}_{s}+\left(v^{-1}-v\right) \sum_{\lambda \in \mathfrak{o}: s \in W_{\lambda}} 1_{\lambda}$ in $H_{\mathfrak{o}}$. It follows that $\tilde{T}_{w}$ is invertible in $H_{\mathfrak{o}}$ for any $w \in W$. As in [7, 31.3], there is a unique ring homomorphism ${ }^{-}: H_{\mathfrak{o}} \rightarrow H_{\mathfrak{o}}$ such that $\bar{T}_{w}=\tilde{T}_{w^{-1}}^{-1}$ for all $w \in W$ and $\overline{v^{m} 1_{\lambda}}=v^{-m} 1_{\lambda}$ for all $\lambda \in \mathfrak{o}$. The square of ${ }^{-}: H_{\mathfrak{o}} \rightarrow H_{\mathfrak{o}}$ is 1 .

Now let $\lambda \in \mathfrak{o}$ and let $\sigma \in W_{\lambda}$ be such that $|\sigma|_{\lambda}=1$. From 1.4(h) we have $\tilde{T}_{\sigma}^{-1} 1_{\lambda}=\tilde{T}_{\sigma} 1_{\lambda}+\left(v^{-1}-v\right) 1_{\lambda}$ so that $\overline{\tilde{T}_{\sigma} 1_{\lambda}} \in H_{\underline{\lambda}}$. Using $1.4(\mathrm{~g}),(\mathrm{h})$ and induction on $|w|_{\lambda}$, we see that for $w \in W_{\lambda}$ we have $\overline{\tilde{T}_{w} 1_{\lambda}} \in H_{\lambda}$. Thus, ${ }^{-}: H_{\mathfrak{o}} \rightarrow H_{\mathfrak{o}}$ restricts to a ring isomorphism ${ }^{-}: H_{\lambda} \rightarrow H_{\lambda}$ whose square is 1 . If $\left(\lambda^{\prime}, z, \lambda\right) \in \Xi$ then $\iota_{z}: H_{\lambda} \rightarrow H_{\lambda^{\prime}}$ is compatible with the bar operators on $H_{\lambda}, H_{\lambda^{\prime}}$. (It is enough to check that for any $\sigma \in W_{\lambda}$ such that $|\sigma|_{\lambda}=1$ we have $\iota_{z}\left(\tilde{T}_{\sigma}^{-1} 1_{\lambda}\right)=\tilde{T}_{z \sigma z^{-1}}^{-1} 1_{\lambda^{\prime}}$; this is immediate.) It follows that the group homomorphism ${ }^{-}: \mathcal{H}^{\bullet} \rightarrow \mathcal{H}^{\bullet}$ whose restriction to any $\mathcal{H}_{\lambda^{\prime}, z, \lambda}^{\bullet}=H_{\lambda}$ is ${ }^{-}: H_{\lambda} \rightarrow H_{\lambda}$ is in fact a ring homomorphism with square 1 . We show that $\theta: \mathcal{H}^{\bullet} \rightarrow H_{\mathfrak{o}}$ is compatible with ${ }^{-}: \mathcal{H}^{\bullet} \rightarrow \mathcal{H}^{\bullet}$ and $^{-}: H_{\mathfrak{o}} \rightarrow H_{\mathfrak{o}}$. It is enough to show that for $\left(\lambda^{\prime}, z, \lambda\right) \in \Xi, w \in W_{\lambda}$, we have $\overline{\tilde{T}_{z} \tilde{T}_{w} 1_{\lambda}}=\tilde{T}_{z} \tilde{T}_{w^{-1}}^{-1} 1_{\lambda}$; it is also enough to show that $\overline{\tilde{T}_{z} 1_{\lambda}}=\tilde{T}_{z} 1_{\lambda}$ or that $\tilde{T}_{z^{-1}}^{-1} 1_{\lambda}=\tilde{T}_{z} 1_{\lambda}$, which follows from 1.4(e).

For $\lambda \in \mathfrak{o},^{-}: H_{\lambda} \rightarrow H_{\lambda}$ corresponds under $\vartheta_{\lambda}$ to a ring isomorphism ${ }^{-}: \mathfrak{H}_{\lambda} \rightarrow \mathfrak{H}_{\lambda}$ such that $\overline{v^{m \mathfrak{T}_{w}}}=v^{-m \mathfrak{T}_{w^{-1}}^{-1}}$ for any $w \in W_{\lambda}, m \in \mathbf{Z}$. Under the isomorphism $\vartheta: \mathcal{H}^{\bullet} \rightarrow \mathcal{H}^{\bullet},{ }^{-}: \mathcal{H}^{\bullet} \rightarrow \mathcal{H}^{\bullet}$ corresponds to a ring isomorphism ${ }^{-}: \mathcal{H}^{\prime} \bullet \mathcal{H}^{\prime \bullet}$ with square 1 ; its restriction to $\mathcal{H}_{\lambda^{\prime}, z, \lambda}^{\prime}=\mathfrak{H}_{\lambda}$ coincides with ${ }^{-}: \mathfrak{H}_{\lambda} \rightarrow \mathfrak{H}_{\lambda}$.

Let $\lambda \in \mathfrak{o}$. By $[6,5.12]$, for any $w \in W_{\lambda}$ there is a unique element $c_{w}^{\lambda} \in \mathfrak{H}_{\lambda}$ such that $\overline{c_{w}^{\lambda}}=c_{w}^{\lambda}$ and $c_{w}^{\lambda}-\mathfrak{T}_{w} \in \sum_{y \in W_{\lambda} ;|y|_{\lambda}<|w|_{\lambda}} v^{-1} \mathbf{Z}\left[v^{-1}\right] \mathfrak{T}_{y}$. Using $\vartheta_{\lambda}$ we deduce that for any $w \in W_{\lambda}$ there is a unique element $c_{w, \lambda} \in H_{\lambda}$ such that $\overline{c_{w, \lambda}}=c_{w, \lambda}$ and $c_{w, \lambda}-\tilde{T}_{w} 1_{\lambda} \in \sum_{y \in W_{\lambda} ;|y|_{\lambda}<|w|_{\lambda}} v^{-1} \mathbf{Z}\left[v^{-1}\right] \tilde{T}_{y} 1_{\lambda}$. 
If $\left(\lambda^{\prime}, z, \lambda\right) \in \Xi$ and $w \in W_{\lambda}$ then $\tilde{T}_{z} c_{w, \lambda} \tilde{T}_{z^{-1}}$ satisfies the definition $c_{z w z^{-1}, \lambda^{\prime}} \in H_{\lambda^{\prime}}$ hence we have $\tilde{T}_{z} c_{w, \lambda} \tilde{T}_{z^{-1}}=c_{z w z^{-1}, \lambda^{\prime}}$.

Now let $w \in W, \lambda \in \mathfrak{o}$. We can write uniquely $w=z \tilde{w}$ where $\tilde{w} \in W_{\lambda}$, $z \in\left[\lambda^{\prime}, \lambda\right]$ for some $\lambda^{\prime} \in \mathfrak{o}$. We set $c_{w, \lambda}=\tilde{T}_{z} c_{\tilde{w}, \lambda}$. We have $\overline{c_{w, \lambda}}=c_{w, \lambda}$ and $c_{w, \lambda}-\tilde{T}_{w} 1_{\lambda} \in \sum_{y \in W_{\lambda} ;|y|_{\lambda}<|\tilde{w}|_{\lambda}} v^{-1} \mathbf{Z}\left[v^{-1}\right] \tilde{T}_{z y} 1_{\lambda}$. It follows that $\left\{c_{w, \lambda} ; w \in\right.$ $W, \lambda \in \mathfrak{o}\}$ is an $\mathcal{A}$-basis of $H_{\mathfrak{o}}$.

1.7. For any $\lambda \in \mathfrak{o}$ we state some properties of the multiplication of elements of form $c_{w, \lambda} \in H_{\lambda}$ with $w \in W_{\lambda}$. These properties follow from the known properties of the multiplication of elements of form $c_{w}^{\lambda} \in \mathfrak{H}_{\lambda}$ via the isomorphism $\vartheta_{\lambda}$. (We use that $W_{\lambda}$ is a Weyl group or an affine Weyl groups, see 1.2(b).) For any $w, w^{\prime}$ in $W_{\lambda}$ we have

$$
c_{w, \lambda} c_{w^{\prime}, \lambda}=\sum_{w^{\prime \prime} \in W_{\lambda}} r_{w, w^{\prime}}^{w^{\prime \prime} ; \lambda} c_{w^{\prime \prime}, \lambda}
$$

where $r_{w, w^{\prime}}^{w^{\prime \prime}} \in \mathcal{A}$ are zero for all but finitely many $w^{\prime \prime}$. Moreover, for any $w^{\prime \prime} \in$ $W_{\lambda}$ there is a well defined smallest integer $a\left(w^{\prime \prime}\right) \geq 0$ such that $v^{a\left(w^{\prime \prime}\right)} r_{w ; w^{\prime}}^{w^{\prime \prime} ; \lambda} \in$ $\mathbf{Z}[v]$ for all $w, w^{\prime}$ in $W_{\lambda}$. We have

$$
r_{w, w^{\prime}}^{w^{\prime \prime} ; \lambda}=\bar{r}_{w ; w^{\prime}}^{w^{\prime \prime} ; \lambda} v^{-a\left(w^{\prime \prime}\right)} \bmod v^{-a\left(w^{\prime \prime}\right)+1} \mathbf{Z}[v]
$$

where $\bar{r}_{w, w^{\prime}}^{w^{\prime \prime} ; \lambda} \in \mathbf{N}$.

We now consider the free abelian group $H_{\lambda}^{\infty}$ with basis $\left\{t_{w} ; w \in W_{\lambda}\right\}$. Then $H_{\lambda}^{\infty}$ is an (associative) ring with multiplication given by

$$
t_{w} t_{w^{\prime}}=\sum_{w^{\prime \prime} \in W_{\lambda}} \bar{r}_{w, w^{\prime}}^{w^{\prime \prime} ; \lambda} t_{w^{\prime \prime}}
$$

It has a unit element of the form $1=\sum_{w \in D_{\lambda}} t_{w}$ where $D_{\lambda}$ is a well defined finite subset of $\left\{w \in W_{\lambda} ; w^{2}=1\right\}$. The $\mathcal{A}$-linear map $\psi_{\lambda}: H_{\lambda} \rightarrow \mathcal{A} \otimes H_{\lambda}^{\infty}$, given by

$$
\psi_{\lambda}\left(c_{w, \lambda}\right)=\sum_{w^{\prime \prime} \in W_{\lambda}, d \in D_{\lambda} ; a(d)=a\left(w^{\prime \prime}\right)} r_{w, d}^{w^{\prime \prime} ; \lambda} t_{w^{\prime \prime}}
$$

is a homomorphism of $\mathcal{A}$-algebras with 1 . 
For $w, w^{\prime}$ in $W_{\lambda}$ we have $w \sim_{\text {left }} w^{\prime}$ (resp. $w \sim_{\text {right }} w^{\prime}$ if and only if for some $u \in W_{\lambda}, t_{w^{\prime}}$ appears with non-zero coefficient in $t_{u} t_{w}$ (resp. in $\left.t_{u} t_{w}\right)$. For $w, w^{\prime}$ in $W_{\lambda}$ we have $w \sim w^{\prime}$ if and only if for some $u, u^{\prime}$ in $W_{\lambda}$ we have $t_{w^{\prime}}$ appears with non-zero coefficient in $t_{u} t_{w} t_{u^{\prime}}$ (or equivalently if for some $w^{\prime \prime} \in W_{\lambda}$ we have $\left.w \sim_{\text {left }} w^{\prime \prime}, w^{\prime} \sim_{\text {right }} w^{\prime \prime}\right)$. Recall from 0.2 that the equivalence classes for $\sim_{\text {left }}$ (resp. $\left.\sim_{\text {right }}, \sim\right)$ are called the left (resp. right, two-sided) cells of $W_{\lambda}$. For any left (resp. right, two-sided) cell $K$ of $W_{\lambda}$ the subgroup $\sum_{w \in K} \mathbf{Z} t_{w}$ is a left (resp. right, two-sided) ideal of $H_{\lambda}^{\infty}$.

Note that the definition of left (resp. right, two-sided) cells of $W_{\lambda}$ depends only on the Coxeter group structure of $W_{\lambda}$ hence these are defined for any Coxeter group $\mathcal{W}$ which is a Weyl group or an affine Weyl group; we shall write $\operatorname{Cell}(\mathcal{W})$ for the set of two-sided cells of $\mathcal{W}$.

Let $\mathcal{J}: \Gamma \rightarrow A l g_{\mathbf{Z}}$ be the functor defined by $\mathcal{J}(\lambda)=H_{\lambda}^{\infty}$ for $\lambda \in \mathfrak{o}$ and the isomorphisms $\iota_{z}: H_{\lambda}^{\infty} \stackrel{\sim}{\rightarrow} H_{\lambda^{\prime}}^{\infty}$ for $\left(\lambda^{\prime}, z, \lambda\right) \in \Xi$ where $\iota_{z}$ maps any basis element $t_{w}\left(w \in W_{\lambda}\right)$ to the basis element $t_{z w z^{-1}}$ of $H_{\lambda^{\prime}}^{\infty}$. Then the (associative) ring $\mathcal{J}^{\bullet}$ is defined as in 1.5.

1.8. For $\left(w_{0}, \lambda_{0}\right),\left(w_{1}, \lambda_{1}\right)$ in $W \times \mathfrak{o}$ we write in $H_{\mathfrak{o}}$ :

$$
c_{w_{0}, \lambda_{0}} c_{w_{1}, \lambda_{1}}=\sum_{\left(w_{2}, \lambda_{2}\right) \in W \times \mathfrak{0}} R_{w_{0}, \lambda_{0} ; w_{1}, \lambda_{1}}^{w_{2}, \lambda_{2}} c_{w_{2}, \lambda_{2}}
$$

where $R_{w_{0}, \lambda_{0} ; w_{1}, \lambda_{1}}^{w_{2}, \lambda_{2}} \in \mathcal{A}$ are zero for all but finitely many $\left(w_{2}, \lambda_{2}\right)$. For $j=$ $0,1,2$ we can write uniquely $w_{j}=z_{j} \tilde{w}_{j}$ where $z_{j} \in\left[\lambda_{j}^{\prime}, \lambda_{j}\right], \tilde{w}_{j} \in W_{\lambda_{j}}$. We have

$$
c_{w_{0}, \lambda_{0}} c_{w_{1}, \lambda_{1}}=\tilde{T}_{z_{0}} c_{\tilde{w}_{0}, \lambda_{0}} \tilde{T}_{z_{1}} c_{\tilde{w}_{1}, \lambda_{1}} .
$$

This is 0 unless $\lambda_{0}=z_{1}\left(\lambda_{1}\right)$. Assume now that $\lambda_{0}=z_{1}\left(\lambda_{1}\right)$. We have

$$
\begin{aligned}
& c_{w_{0}, \lambda_{0}} c_{w_{1}, \lambda_{1}}=\tilde{T}_{z_{0}} \tilde{T}_{z_{1}} c_{z_{1}^{-1}} \tilde{w}_{0} z_{1}, \lambda_{1} c_{\tilde{w}_{1}, \lambda_{1}}
\end{aligned}
$$

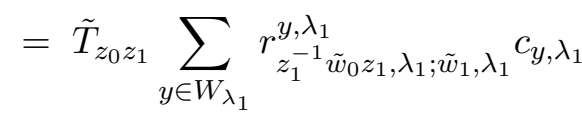

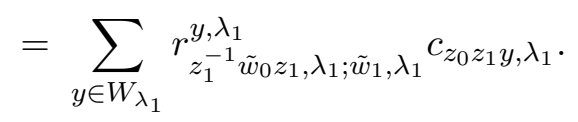


We see that $R_{w_{0}, \lambda_{0} ; w_{1}, \lambda_{1}}^{w_{2}, \lambda_{2}}=0$ unless the conditions $\lambda_{2}=\lambda_{1}, \lambda_{0}=z_{1}\left(\lambda_{1}\right), z_{2}=$ $z_{0} z_{1}$ are satisfied; if these conditions are satisfied, then

$$
R_{w_{0}, \lambda_{0} ; w_{1}, \lambda_{1}}^{w_{2}, \lambda_{2}}=r_{z_{1}^{-1} \tilde{w}_{0} z_{1}, \tilde{w}_{1}}^{\tilde{w}_{2} ; \lambda_{1}}
$$

We see that

(a) $v^{a_{w_{2}, \lambda_{2}}} R_{w_{0}, \lambda_{0} ; w_{1}, \lambda_{1}}^{w_{2}, \lambda_{2}} \in \mathbf{Z}[v]$

where $a_{w_{2}, \lambda_{2}}=a_{\tilde{w}_{2}}$ is defined in terms of $W_{\lambda_{2}}$ and that $a_{w_{2}, \lambda_{2}}$ is the smallest integer such that (a) holds for any $\left(w_{0}, \lambda_{0}\right),\left(w_{1}, \lambda_{1}\right)$. We now define $\bar{R}_{w_{0}, \lambda_{0} ; w_{1}, \lambda_{1}}^{w_{2}, \lambda_{2}} \in \mathbf{Z}$ by

$$
R_{w_{0}, \lambda_{0} ; w_{1}, \lambda_{1}}^{w_{2}, \lambda_{2}}=\bar{R}_{w_{0}, \lambda_{0} ; w_{1}, \lambda_{1}}^{w_{2}, \lambda_{2}} v^{-a\left(w_{2}, \lambda_{2}\right)} \bmod v^{-a\left(w_{2}, \lambda_{2}\right)+1} \mathbf{Z}[v] .
$$

We consider the free abelian group $\mathfrak{H}_{\mathfrak{o}}^{\infty}$ with basis $\left\{t_{w, \lambda} ; w \in W, \lambda \in \mathfrak{o}\right\}$. We define a ring structure on $\mathfrak{H}_{\mathfrak{o}}^{\infty}$ by

$$
t_{w_{0}, \lambda_{0}} t_{w_{1}, \lambda_{1}}=\sum_{\left(w_{2}, \lambda_{2}\right) \in W \times \mathfrak{o}} \bar{R}_{w_{0}, \lambda_{0} ; w_{1}, \lambda_{1}}^{w_{2}, \lambda_{2}} t_{w_{2}, \lambda_{2}},
$$

or equivalently (for $\tilde{w}_{0} \in W_{\lambda_{0}}, \tilde{w}_{1} \in W_{\lambda_{1}}, z_{0} \in\left[\lambda_{0}^{\prime}, \lambda_{0}\right], z_{1} \in\left[\lambda_{1}^{\prime}, \lambda_{1}\right]$ ):

$$
t_{z_{0} \tilde{w}_{0}, \lambda_{0}} t_{z_{1} \tilde{w}_{1}, \lambda_{1}}=\sum_{\tilde{w}_{2} \in W_{\lambda_{1}}} \bar{r}_{z_{1}^{-1} \tilde{w}_{0} z_{1}, \tilde{w}_{1}}^{\tilde{w}_{2} \lambda_{1}} t_{z_{0} z_{1} \tilde{w}_{2}, \lambda_{1}},
$$

if $\lambda_{0}=z_{1}\left(\lambda_{1}\right)$,

$$
t_{z_{0} \tilde{w}_{0}, \lambda_{0}} t_{z_{1} \tilde{w}_{1}, \lambda_{1}}=0 \text {, if } \lambda_{0} \neq z_{1}\left(\lambda_{1}\right) .
$$

This ring is associative. Indeed, this ring has the same multiplication rule as the ring $\mathcal{J}^{\bullet}$ in 1.7, which is known to be associative from 1.5. Note that the ring $\mathfrak{H}_{\mathfrak{o}}^{\infty}$ has a unit element, namely $\sum_{\lambda \in \mathfrak{o}} \sum_{w \in D_{\lambda}} t_{w, \lambda}$.

The $\mathcal{A}$-algebra homomorphisms $\psi_{\lambda}: H_{\lambda} \rightarrow \mathcal{A} \otimes H_{\lambda}^{\infty}$ combine to give an $\mathcal{A}$-algebra homomorphism $\psi: \mathcal{H}^{\bullet} \rightarrow \mathcal{A} \otimes \mathcal{J}^{\bullet}$ or equivalently $\psi: H_{\mathfrak{o}} \rightarrow$ $\mathcal{A} \otimes \mathfrak{H}_{\mathfrak{o}}^{\infty} ;$ it is given by

$$
\psi\left(c_{w, \lambda}\right)=\sum_{\left(w_{2}, \lambda_{2}\right) \in W \times \mathfrak{o}, \lambda_{1} \in \mathfrak{o}, d \in D_{\lambda_{1}} ; a\left(w_{2}, \lambda_{2}\right)=a\left(d, \lambda_{1}\right)} R_{w, \lambda ; d, \lambda_{1}}^{w_{2}, \lambda_{2}} t_{w_{2}, \lambda_{2}}
$$


that is,

$$
\psi\left(c_{w, \lambda}\right)=\sum_{\left(\tilde{w}_{2} \in W_{\lambda}, d \in D_{\lambda} ; a\left(\tilde{w}_{2}\right)=a(d)\right.} r_{\tilde{w}, d}^{\tilde{w}_{2} ; \lambda} t_{z \tilde{w}_{2}, \lambda}
$$

where $w=z \tilde{w}$ with $\tilde{w} \in W_{\lambda}, z \in[w(\lambda), \lambda]$.

1.9. We now describe the left (resp. right) cells of $W \times \mathfrak{o}$ defined in terms of the basis $\left(t_{w, \lambda}\right)$ of $H_{\mathfrak{o}}^{\infty}$. (See 0.2.) Let $(w, \lambda),\left(w^{\prime}, \lambda^{\prime}\right)$ in $W \times \mathfrak{o}$. We say that $(w, \lambda) \approx_{\text {left }}\left(w^{\prime}, \lambda^{\prime}\right)$ (resp. $\left.(w, \lambda) \approx_{\text {right }}\left(w^{\prime}, \lambda^{\prime}\right)\right)$ if for some $\left(u, \lambda_{1}\right) \in$ $W \times \mathfrak{o}, t_{w^{\prime}, \lambda^{\prime}}$ appears with non-zero coefficient in the product $t_{u, \lambda_{1}} t_{w, \lambda}$ (resp. $\left.t_{w, \lambda} t_{u, \lambda_{1}}\right)$.

We write $w=z \tilde{w}, w^{\prime}=z^{\prime} \tilde{w}^{\prime}$ where $\tilde{w} \in W_{\lambda}, \tilde{w}^{\prime} \in W_{\lambda^{\prime}}, z \in[w(\lambda), \lambda]$, $z^{\prime} \in\left[w^{\prime}\left(\lambda^{\prime}\right), \lambda^{\prime}\right]$. From $1.8(\mathrm{~b}),(\mathrm{c})$ we see that the condition that $(w, \lambda) \approx_{\text {left }}$ $\left(w^{\prime}, \lambda^{\prime}\right)$ is that $\lambda=\lambda^{\prime}$ and $\tilde{w} \sim_{l e f t} \tilde{w}^{\prime}$ in $W_{\lambda}=W_{\lambda^{\prime}}$; the condition that $(w, \lambda) \approx_{\text {right }}\left(w^{\prime}, \lambda^{\prime}\right)$ is that $\lambda=\left(z^{-1} z^{\prime}\right)\left(\lambda^{\prime}\right)$ and $\tilde{w}^{\prime} \sim_{\text {right }}\left(z^{\prime-1} z\right) \tilde{w}\left(z^{-1} z^{\prime}\right)$ in $W_{\lambda^{\prime}}=W_{\left(z^{\prime-1} z\right)(\lambda)}$.

Using the results in 1.7 we deduce that $\approx_{\text {left }}$ and $\approx_{\text {right }}$ are equivalence relations on $W \times \mathfrak{o}$. In particular, $\approx_{l e f t}$ is transitive, hence if for $(w, \lambda) \in$ $W \times \mathfrak{o}$ we set $\widetilde{(w, \lambda)}=\left\{\left(w^{\prime}, \lambda^{\prime}\right) \in W \times \mathfrak{o} ;(w, \lambda) \approx_{\text {left }}\left(w^{\prime}, \lambda^{\prime}\right)\right\}$, then the subgroup spanned by $\left\{t_{w^{\prime}, \lambda^{\prime}} ;\left(w^{\prime}, \lambda^{\prime}\right) \in \widehat{(w, \lambda)}\right\}$ is a left ideal of $H_{\mathfrak{o}}^{\infty}$, so that $\widetilde{(w, \lambda)}$ is a union of left cells. If $(w, \lambda) \approx_{\text {left }}\left(w^{\prime}, \lambda^{\prime}\right)$, then clearly any left ideal of $H_{\mathfrak{o}}^{\infty}$ spanned by a subset of the canonical basis of $H_{\mathfrak{o}}^{\infty}$ and containing $t_{w, \lambda}$ must also contain $t_{w^{\prime}, \lambda^{\prime}}$. Since $\approx_{l e f t}$ is symmetric we must also have $\left(w^{\prime}, \lambda^{\prime}\right) \approx_{\text {left }}(w, \lambda)$ hence any left ideal of $H_{\mathfrak{o}}^{\infty}$ spanned by a subset of the canonical basis of $H_{\mathfrak{o}}^{\infty}$ and containing $t_{w^{\prime}, \lambda^{\prime}}$ must also contain $t_{w, \lambda}$; it follows that $(w, \lambda),\left(w^{\prime}, \lambda^{\prime}\right)$ are in the same left cell. We now see that any equivalence class for $\approx_{l e f t}$ is exactly one left cell. We see that the canonical basis elements indexed by any left cell span a left ideal. The same argument shows that the equivalence classes for $\approx_{\text {right }}$ are exactly the right cells and that the canonical basis elements indexed by any right cell span a right ideal.

We now see that we have a natural bijection

\{left cells in $W \times \mathfrak{o}\} \leftrightarrow\left\{(\lambda, \gamma) ; \lambda \in \mathfrak{o}, \gamma=\right.$ left cell in $\left.W_{\lambda}\right\}$

The bijection associates to each $(\lambda, \gamma)$ in the right hand side the left cell $\left\{(z \tilde{w}, \lambda) ; \tilde{w} \in \gamma, z=\min \left(z W_{\lambda}\right)\right\}$ of $W \times \mathfrak{o}$. We also see that the right cells in 
$W \times \mathfrak{o}$ are the images of the left cells under the involution $W \times \mathfrak{o} \rightarrow W \times \mathfrak{o}$, $(w, \lambda) \mapsto\left(w^{-1}, w(\lambda)\right)$.

1.10. We now describe the two-sided cells of $W \times \mathfrak{o}$ defined by the basis $\left(t_{w, \lambda}\right)$ of $H_{\mathfrak{o}}^{\infty}$. (See 0.2). Let $\lambda, \lambda^{\prime}$ in $\mathfrak{o}$; let $w, w^{\prime}$ in $W$.

We say that $(w, \lambda) \approx\left(w^{\prime}, \lambda^{\prime}\right)$ if for some $\left(u_{1}, \lambda_{1}\right),\left(u_{2}, \lambda_{2}\right)$ in $W \times \mathfrak{o}, t_{w^{\prime}, \lambda^{\prime}}$ appears with non-zero coefficient in the product $t_{u_{1}, \lambda_{1}} t_{w, \lambda} t_{u_{2}, \lambda_{2}}$ or equivalently, if for some $\left(w^{\prime \prime}, \lambda^{\prime \prime}\right)$ we have $(w, \lambda) \approx_{\text {left }}\left(w^{\prime \prime}, \lambda^{\prime \prime}\right)$ and $\left(w^{\prime}, \lambda^{\prime}\right) \approx_{\text {right }}$ $\left(w^{\prime \prime}, \lambda^{\prime \prime}\right)$. (The equivalence uses the positivity of the structure constants of $H_{\mathfrak{o}}^{\infty}$.) We write $w=z \tilde{w}, w^{\prime}=z^{\prime} \tilde{w}^{\prime}$ where $\tilde{w} \in W_{\lambda}, \tilde{w}^{\prime} \in W_{\lambda^{\prime}}, z \in[w(\lambda), \lambda]$, $z^{\prime} \in\left[w^{\prime}\left(\lambda^{\prime}\right), \lambda^{\prime}\right]$. From 1.9 we see that the condition that $(w, \lambda) \approx\left(w^{\prime}, \lambda^{\prime}\right)$ is that for some $\lambda^{\prime \prime} \in \mathfrak{o}, \tilde{w}^{\prime \prime} \in W_{\lambda^{\prime \prime}}, z_{1} \in\left[\lambda^{\prime}, \lambda^{\prime \prime}\right]$ we have $\lambda=\lambda^{\prime \prime}, \tilde{w} \sim_{\text {left }} \tilde{w}^{\prime \prime}$ in $W_{\lambda}=W_{\lambda^{\prime \prime}}, \tilde{w}^{\prime \prime} \sim_{\text {right }} z_{1}^{-1} \tilde{w}^{\prime} z_{1}$ in $W_{\lambda^{\prime \prime}}=W_{z_{1}^{-1}\left(\lambda^{\prime}\right)}$ or equivalently: for some $z_{1} \in\left[\lambda^{\prime}, \lambda\right]$ we have $\tilde{w} \sim z_{1}^{-1} \tilde{w}^{\prime} z_{1}$ in $W_{\lambda}=W_{z_{1}^{-1}\left(\lambda^{\prime}\right)}$. Using the results in 1.7 we deduce that $\approx$ is an equivalence relation on $W \times \mathfrak{o}$.

In particular, $\approx$ is transitive, hence if for $(w, \lambda) \in W \times \mathfrak{o}$ we set $(\hat{w, \lambda})=$ $\left\{\left(w^{\prime}, \lambda^{\prime}\right) \in W \times \mathfrak{o} ;(w, \lambda) \approx\left(w^{\prime}, \lambda^{\prime}\right)\right\}$, then the subgroup spanned by $\left\{t_{w^{\prime}, \lambda^{\prime}}\right.$; $\left.\left(w^{\prime}, \lambda^{\prime}\right) \in(\hat{w, \lambda})\right\}$ is a two-sided ideal of $H_{\mathfrak{o}}^{\infty}$, so that $(\hat{w, \lambda})$ is a union of two-sided cells.

Now assume that $(w, \lambda) \approx\left(w^{\prime}, \lambda^{\prime}\right)$; let $\left(w^{\prime \prime}, \lambda^{\prime \prime}\right)$ be such that $(w, \lambda) \approx_{\text {left }}$ $\left(w^{\prime \prime}, \lambda^{\prime \prime}\right)$ and $\left(w^{\prime}, \lambda^{\prime}\right) \approx_{\text {right }}\left(w^{\prime \prime}, \lambda^{\prime \prime}\right)$. By 1.9 , we have $(w, \lambda) \sim_{\text {left }}\left(w^{\prime \prime}, \lambda^{\prime \prime}\right)$ and $\left(w^{\prime}, \lambda^{\prime}\right) \sim_{\text {right }}\left(w^{\prime \prime}, \lambda^{\prime \prime}\right)$. Hence if $\mathcal{I}$ is a two-sided ideal of $H_{\mathfrak{o}}^{\infty}$ spanned by a subset of the canonical basis then we have $t_{w, \lambda} \in \mathcal{I} \leftrightarrow t_{w^{\prime \prime}, \lambda^{\prime \prime}} \in \mathcal{I} \leftrightarrow$ $t_{w^{\prime}, \lambda^{\prime}} \in \mathcal{I}$. It follows that $(w, \lambda) \sim\left(w^{\prime}, \lambda^{\prime}\right)$. We see that any equivalence class for $\approx$ is contained in a two-sided cell. As we have seen earlier, any equivalence class for $\approx$ is a union of two-sided cells, hence it is exactly one two-sided cell. Also, the canonical basis elements indexed by any equivalence class for $\approx$ (hence by any two-sided cell) span a two-sided ideal of $H_{\mathfrak{o}}^{\infty}$.

Let $\operatorname{Cell}(W \times \mathfrak{o})$ be the set of two-sided cells of $W \times \mathfrak{o}$. Note that for any $\lambda \in \mathfrak{o}$, the group $[\lambda, \lambda]$ acts by conjugation on $W_{\lambda}$; this action induces an action of $[\lambda, \lambda]$ on $\operatorname{Cell}\left(W_{\lambda}\right)$. If $\Delta \in \operatorname{Cell}(W \times \mathfrak{o})$, for any $\lambda \in \mathfrak{o}$ we set $\Delta_{\lambda}=\left\{\tilde{w} \in W_{\lambda} ;(\tilde{w}, \lambda) \in \Delta\right\}$; this is a union of the two-sided cells of $W_{\lambda}$ in a fixed $[\lambda, \lambda]$-orbit. Moreover, if $\lambda, \lambda^{\prime}$ are elements of $\mathfrak{o}$ then $z \Delta_{\lambda} z^{-1}=D_{\lambda^{\prime}}$ for any $z \in\left[\lambda^{\prime}, \lambda\right]$. We now see that for each $\lambda \in \mathfrak{o}$ we have a bijection $\Delta \mapsto \Delta_{\lambda}$ 
between $\operatorname{Cell}(W \times \mathfrak{o})$ and the set of orbits of the conjugation of $[\lambda, \lambda]$ on $\operatorname{Cell}\left(W_{\lambda}\right)$.

From the results in 1.8 we see that if $\Delta, \lambda$ are as above, then the function $(w, \lambda) \mapsto a(w, \lambda)$ on $\Delta$ is constant with value equal to the value of the $a$ function on any of the two-sided cells of $W_{\lambda}$ contained in $\Delta_{\lambda}$.

1.11. Let $H=\oplus_{\mathfrak{o}} H_{\mathfrak{o}}$ (resp. $H^{\infty}=\oplus_{\mathfrak{o}} H_{\mathfrak{o}}^{\infty}$ ) where $\mathfrak{o}$ runs over all $W$-orbits in $\bar{V}_{0}^{\prime}$. We view $H$ (resp. $H^{\infty}$ ) as an $\mathcal{A}$-algebra (resp. ring) without 1 in general) in which $H_{\mathfrak{o}}$ (resp. $H_{\mathfrak{o}}^{\infty}$ ) is a subalgebra (resp. subring) for any $\mathfrak{o}$ and $H_{\mathfrak{o}} H_{\mathfrak{o}^{\prime}}=0$ (resp. $H_{\mathfrak{o}}^{\infty} H_{\mathfrak{o}^{\prime}}^{\infty}=0$ ) for $\mathfrak{o} \neq \mathfrak{o}^{\prime}$. Note that $\left\{c_{w, \lambda} ; w \in W, \lambda \in \bar{V}_{0}^{\prime}\right\}$ is an $\mathcal{A}$-basis of $H$ and that $\left\{t_{w, \lambda} ; w \in W, \lambda \in \bar{V}_{0}^{\prime}\right\}$ is a $\mathbf{Z}$-basis of $H^{\infty}$. The left (resp. right, two-sided) cells of $W \times \bar{V}_{0}^{\prime}$ are defined in terms of the basis $\left(t_{w, \lambda}\right)$ of $H^{\infty}$ as in 0.2 . Using 1.9 we have a natural bijection

$$
\text { \{left cells in } \left.W \times \bar{V}_{0}^{\prime}\right\} \leftrightarrow\left\{(\lambda, \gamma) ; \lambda \in \bar{V}_{0}^{\prime}, \gamma=\text { left cell in } W_{\lambda}\right\}
$$

The bijection associates to each $(\lambda, \gamma)$ in the right hand side the left cell $\left\{(z \tilde{w}, \lambda) ; \tilde{w} \in \gamma, z=\min \left(z W_{\lambda}\right)\right\}$ of $W \times \bar{V}_{0}^{\prime}$. The right cells in $W \times \bar{V}_{0}^{\prime}$ are the images of the left cells under the involution $W \times \bar{V}_{0}^{\prime} \rightarrow W \times \bar{V}_{0}^{\prime}$, $(w, \lambda) \mapsto\left(w^{-1}, w(\lambda)\right)$. Let $\operatorname{Cell}\left(W \times \bar{V}_{0}^{\prime}\right)$ be the set of two-sided cells of $W \times \bar{V}_{0}^{\prime}$. We have $\operatorname{Cell}\left(W \times \bar{V}_{0}^{\prime}\right)=\sqcup_{\mathfrak{o}} \operatorname{Cell}(W \times \mathfrak{o})$ where $\mathfrak{o}$ runs over all $W$-orbits in $\bar{V}_{0}^{\prime}$ and $\operatorname{Cell}(W \times \mathfrak{o})$ is described in 1.10 .

Let $\left(w^{\prime \prime}, \lambda^{\prime \prime}\right) \in W \times \bar{V}_{0}^{\prime}$. There is a well defined smallest integer $a(w, \lambda) \geq$ 0 such that for any $(w, \lambda),\left(w^{\prime}, \lambda^{\prime}\right)$ in $W \times \bar{V}_{0}^{\prime}$, the coefficient of $c_{w^{\prime \prime}, \lambda^{\prime \prime}}$ in the product $c_{w, \lambda} c_{w^{\prime}, \lambda^{\prime}}$ belongs to $v^{-a\left(w^{\prime \prime}, \lambda^{\prime \prime}\right)} \mathbf{Z}[v]$. From 1.10 we see that for any $\Delta \in \operatorname{Cell}\left(W \times \bar{V}_{0}^{\prime}\right)$ the function $\Delta \rightarrow \mathbf{N},\left(w^{\prime \prime}, \lambda^{\prime \prime}\right) \mapsto a\left(w^{\prime \prime}, \lambda^{\prime \prime}\right)$ is constant on $\Delta$.

\section{Conjugacy Classes}

2.1. Let $\mathcal{W}$ be an affine Weyl group. Let $\mathcal{T}$ be the set of translations in $\mathcal{W}$, that is the set of the elements of $\mathcal{W}$ whose $\mathcal{W}$-conjugacy class is finite. Note that $\mathcal{T}$ is a subgroup of finite index in $\mathcal{W}$. Let $\mathcal{S}$ be the set of simple reflections of $\mathcal{W}$. Let $A(\mathcal{W})$ be the group of automorphisms $\sigma: \mathcal{W} \rightarrow \mathcal{W}$ such that $\sigma(\mathcal{S})=\mathcal{S}$; note that $\sigma \in A(\mathcal{W})$ is uniquely determined by its restriction to $\mathcal{S}$. 
We now assume that the affine Weyl group $\mathcal{W}$ is irreducible. Let $\mathcal{S}_{0}$ be the set of all $s \in \mathcal{S}$ such that $\mathcal{S}-\{s\}$ together with $\mathcal{T}$ generates $\mathcal{W}$. We have $\mathcal{S}_{0} \neq \emptyset$. If $\sigma \in A(\mathcal{W})$ then $\sigma$ restricts to a permutation of $\mathcal{S}_{0}$. Let $A_{0}(\mathcal{W})$ be the set of all $\sigma \in A(\mathcal{W})$ such that $\sigma: \mathcal{S}_{0} \rightarrow \mathcal{S}_{0}$ is either fixed point free or the identity. Note that $A_{0}(\mathcal{W})$ is a normal subgroup of $A(\mathcal{W})$; it acts simply transitively on $\mathcal{S}_{0}$. For any $s \in \mathcal{S}_{0}$ let $A^{s}(\mathcal{W})$ be the set of all $\sigma \in A(\mathcal{W})$ such that $\sigma(s)=s$ (a subgroup of $A(\mathcal{W})$ ); we have $A(\mathcal{W})=A_{0}(\mathcal{W}) A^{s}(\mathcal{W})$, $A_{0}(\mathcal{W}) \cap A^{s}(\mathcal{W})=\{1\}$.

2.2. The results in this subsection can be deduced from those in 2.1. Let $\mathcal{W}$ be an affine Weyl group with set of simple reflections $\mathcal{S}$. We can write $\mathcal{W}=\prod_{u \in \mathcal{U}} \mathcal{W}^{u}, \mathcal{S}=\sqcup_{u \in \mathcal{U}} \mathcal{S}^{u}$ where $\mathcal{W}^{u}(u \in \mathcal{U})$ is a finite collection of irreducible affine Weyl groups and $\mathcal{S}^{u}$ is the set of simple reflections of $\mathcal{W}^{u}$. We set $\mathcal{S}_{0}=\sqcup_{u \in \mathcal{U}} \mathcal{S}_{0}^{u}$ where $\mathcal{S}_{0}^{u} \subset \mathcal{S}^{u}$ is as in 2.1 (with $\mathcal{W}, \mathcal{S}$ replaced by $\left.\mathcal{W}^{u}, \mathcal{S}^{u}\right)$. We define $A(\mathcal{W})$ as in 2.1. Let $A_{0}(\mathcal{W})$ be the set of all $\sigma \in A(\mathcal{W})$ such that for any $u \in \mathcal{U}$, we have $\sigma\left(\mathcal{W}^{u}\right)=\mathcal{W}^{u}$ and $\left.\sigma\right|_{\mathcal{W}^{u}} \in A_{0}\left(\mathcal{W}^{u}\right)$. Let $\mathbf{s}$ be a subset of $\mathcal{S}_{0}$ such that for any $u \in \mathcal{U}$, s contains exactly one element of $\mathcal{S}_{0}^{u}$. (Note that $A_{0}(\mathcal{W})$ acts simply transitively on the set of all such s.) Let $A^{\mathbf{s}}(\mathcal{W})$ be the set of all $\sigma \in A(\mathcal{W})$ such that $\sigma(\mathbf{s})=\mathbf{s}$ (a subgroup of $A(\mathcal{W})$ ); we have $A(\mathcal{W})=A_{0}(\mathcal{W}) A^{\mathrm{s}}(\mathcal{W}), A_{0}(\mathcal{W}) \cap A^{\mathrm{s}}(\mathcal{W})=\{1\}$.

2.3. In the setup of 2.2 let $\sigma \in A(\mathcal{W})$. Let $\mathcal{T}$ be as in 2.1. The following property (which is checked case by case) has been stated in [2]:

(a) we have $\sigma \in A_{0}(\mathcal{W})$ if and only if there exists $w \in \mathcal{W}$ such that $\sigma(t)=$ $w t w^{-1}$ for all $w \in W$.

Note that the image of $w$ in $\mathcal{W} / \mathcal{T}$ is uniquely determined by $\sigma$. In particular, $\sigma \mapsto w \mathcal{T}$ is a well defined (injective) homomorphism $A_{0}(\mathcal{W}) \rightarrow$ $\mathcal{W} / \mathcal{T}$. We show:

(b) If $\sigma \in A_{0}(\mathcal{W})$ and $c \in \operatorname{Cell}(\mathcal{W})$, then $\sigma(c)=c$.

This is proved in [4, 4.9(b)] using the property (a) of $\sigma$.

2.4. In this subsection we assume that we are in case B. Let $\lambda \in \bar{V}_{0}^{\prime}$. Recall that $\underline{R}_{\lambda}=\{h \in \underline{R} ;(h, \lambda)=0 \in \mathbf{Q} / \mathbf{Z}\}, R_{\lambda}=\left\{h+m \delta_{h} c \in R ; h \in\right.$ 
$\left.\underline{R}_{\lambda}, m \in \mathbf{Z}\right\}$. Let $\underline{R}_{\lambda}^{u}(u \in \mathcal{U})$ be the irreducible components of $\underline{R}_{\lambda}$. Note that $\underline{R}_{\lambda}^{+}=\underline{R}_{\lambda} \cap V^{+}$is a set of positive (co)roots for $\underline{R}_{\lambda}$ with set of simple (co)roots $\underline{\Pi}_{\lambda}=\left\{h^{u, j} ; u \in \mathcal{U}, j \in\left[1, t_{u}\right]\right\} \subset \underline{R}_{\lambda}^{+}$(notation of 1.3). Let ' $\underline{\Pi}_{\lambda}=\left\{h^{u, 0} ; u \in\right.$ $\mathcal{U}\} \subset R_{\lambda}$ (notation of 1.3), ' $\Pi_{\lambda}=\left\{h^{u, 0}+\delta_{h^{u, 0}} c ; u \in \mathcal{U}\right\} \subset R_{\lambda}^{+}, \hat{\Pi}_{\lambda}=$ $\underline{\Pi}_{\lambda} \sqcup^{\prime} \underline{\Pi}_{\lambda} \subset R_{\lambda}, \Pi_{\lambda}=\underline{\Pi}_{\lambda} \sqcup^{\prime} \Pi_{\lambda} \subset R_{\lambda}^{+}$. Note that we have a unique bijection $\Pi_{\lambda} \sim \tilde{\Pi}_{\lambda}, \chi \mapsto \hat{\chi}$, such that for $\chi \in \Pi_{\lambda}$ we have $\hat{\chi} \in \chi+\mathbf{Z} c$. Recall from 1.3 that $W_{\lambda}$ is an affine Weyl group with Coxeter generators $\mathcal{S}_{\lambda}=\left\{s_{\chi} ; \chi \in \Pi_{\lambda}\right\}$. The results of 2.2, 2.3 are applicable with $(\mathcal{W}, \mathcal{S}, \mathcal{T})=\left(W_{\lambda}, \mathcal{S}_{\lambda}, \mathcal{Q}\right)$. Let $\underline{W}_{\lambda}$ be the subgroup of $\underline{W}$ generated by $\left\{s_{\chi} ; \chi \in \underline{R}_{\lambda}\right\}$ (a subgroup of $W_{\lambda}$. Note that $\underline{W}_{\lambda}$ is a Weyl group with Coxeter generators $\left\{s_{h} ; h \in \underline{\Pi}_{\lambda}\right\}$. Let $\underline{W}_{\lambda}^{\prime}=\underline{W} \cap W_{\lambda}^{\prime}=\{w \in \underline{W} ; w(\lambda)=\lambda\}$. We have $\underline{W}_{\lambda} \subset \underline{W}_{\lambda}^{\prime}$. We write $\Omega_{\lambda}$ instead of

$$
[\lambda, \lambda]=\left\{z \in W_{\lambda}^{\prime} ; z\left(R_{\lambda}^{+}\right)=R_{\lambda}^{+}\right\}=\left\{z \in W_{\lambda}^{\prime} ; z\left(\Pi_{\lambda}\right)=\Pi_{\lambda}\right\}
$$

Let

$$
\underline{\Omega}_{\lambda}=\left\{z \in \underline{W}_{\lambda}^{\prime} ; z\left(\underline{R}_{\lambda}^{+}\right)=\underline{R}_{\lambda}^{+}\right\}=\left\{z \in \underline{W}_{\lambda}^{\prime} ; z\left(\underline{\Pi}_{\lambda}\right)=\underline{\Pi}_{\lambda}\right\} .
$$

We have $\underline{W}_{\lambda}^{\prime}=\underline{\Omega}_{\lambda} \underline{W}_{\lambda}$ and $\underline{\Omega}_{\lambda} \cap \underline{W}_{\lambda}=\{1\}$. We show:

(a) $\underline{\Omega}_{\lambda} \subset \Omega_{\lambda}$.

Let $z \in \underline{\Omega}_{\lambda}$. Let $u \in \mathcal{U}$. For some $u^{\prime} \in \mathcal{U}, h \mapsto z(h)$ defines an isomorphism $\underline{R}_{\lambda}^{u} \widetilde{\sim} \underline{R}_{\lambda}^{u^{\prime}}$ which takes simple roots of $\underline{R}_{\lambda}^{u}$ to simple roots of $\underline{R}_{\lambda}^{u^{\prime}}$; hence it takes $h^{u, 0}$ to $h^{u^{\prime}, 0}$ and we must have $\delta_{h^{u, 0}}=\delta_{h^{u^{\prime}, 0}}$. We have also $z(c)=c$, since $z \in \underline{W}$. Thus we have $z\left(h^{u, 0}+\delta_{h^{u, 0}} c\right)=h^{u^{\prime}, 0}+\delta_{h^{u^{\prime}, 0}} c$. We see that $z\left({ }^{\prime} \Pi_{\lambda}\right) \subset{ }^{\prime} \Pi_{\lambda}$, hence $z\left({ }^{\prime} \Pi_{\lambda}\right)={ }^{\prime} \Pi_{\lambda}$. We see that $z\left(\Pi_{\lambda}\right)=\Pi_{\lambda}$ so that $z \in \Omega_{\lambda}$. This proves (a).

Let $\mathfrak{A}_{\lambda}$ be the group of permutations $\rho: \Pi_{\lambda} \rightarrow \Pi_{\lambda}$ with the following property: there exists (a necessarily unique) $\sigma=\sigma_{\rho} \in A\left(W_{\lambda}\right)$ such that $\sigma\left(s_{\chi}\right)=s_{\rho(\chi)}$ for any $\chi \in \Pi_{\lambda}$. Note that $\rho \mapsto \sigma_{\rho}$ is an isomorphism $\mathfrak{A}_{\lambda} \stackrel{\sim}{\rightarrow} A\left(W_{\lambda}\right)$. Each $\rho \in \mathfrak{A}_{\lambda}$ defines a permutation $\hat{\rho}: \hat{\Pi}_{\lambda} \rightarrow \hat{\Pi}_{\lambda}$ by $\hat{\rho}(\hat{\chi})=\widehat{\rho(\chi)}$ for any $\chi \in \Pi_{\lambda}$. Now $\rho \mapsto \hat{\rho}$ defines an isomorphism of $\mathfrak{A}_{\lambda}$ onto a subgroup $\hat{\mathfrak{A}}_{\lambda}$ of the group of permutations of $\hat{\Pi}_{\lambda}$. Let $\tilde{\Omega}_{\lambda}$ be the set of all $z \in \underline{W}_{\lambda}^{\prime}$ such that $z\left(\hat{\Pi}_{\lambda}\right)=\hat{\Pi}_{\lambda}$ and the permutation of $\hat{\Pi}_{\lambda}$ defined by $h \mapsto z(h)$ belongs to $\hat{\mathfrak{A}}_{\lambda}$. 
Note that any element $w \in W$ can be written uniquely in the form $w=t \underline{w}$ where $\underline{w} \in \underline{W}, t \in \mathcal{Q}$; moreover $w \mapsto \underline{w}$ is a homomorphism $\tilde{k}: W \rightarrow \underline{W}$ with kernel $\mathcal{Q}$. We show:

(b) we have $\tilde{k}\left(\Omega_{\lambda}\right) \subset \tilde{\Omega}_{\lambda}$ hence $\tilde{k}$ defines a homomorphism $k: \Omega_{\lambda} \rightarrow \tilde{\Omega}_{\lambda}$ with kernel contained in $\mathcal{Q}$.

Let $w \in \Omega_{\lambda}$. We write $w=t \underline{w}$ with $\underline{w} \in \underline{W}, t \in \mathcal{Q}$. Since $\lambda \in \bar{V}_{0}^{\prime}$, we have $t(\lambda)=\lambda$. Thus $\underline{w}(\lambda)=t^{-1} w(\lambda)=t^{-1}(\lambda)=\lambda$, so that $\underline{w} \in \underline{W}_{\lambda}^{\prime}$. Now let $\chi \in \Pi_{\lambda}$ so that $\hat{\chi} \in \hat{\Pi}_{\lambda}$ and $w(\chi) \in \Pi_{\lambda}$. We show that $\underline{w}(\hat{\chi})=\widehat{w(\chi)}$. Now $\underline{w}(\hat{\chi}) \in \underline{R}$ and $h=\widehat{w(\chi)}$ is the unique element in $\underline{R}$ such that $w(\chi)-h \in \mathbf{Z} c$. Thus it is enough to show that $w(\chi)-\underline{w}(\hat{\chi}) \in \mathbf{Z} c$. We have $\chi=\hat{\chi}+m c$ with $m \in \mathbf{Z}$ hence it is enough to show that $w(\hat{\chi}+m c)-\underline{w}(\hat{\chi}) \in \mathbf{Z} c$. Since $\underline{w}(c)=c$ and $w=t \underline{w}$, it is enough to show that $t \underline{w}(\hat{\chi})-\underline{w}(\hat{\chi}) \in \mathbf{Z} c$ or, setting $h^{\prime}=\underline{w}(\hat{\chi}) \in \underline{R}$, that $t\left(h^{\prime}\right)-h^{\prime} \in \mathbf{Z} c$. The last identity holds for any $t \in \mathcal{Q}, h^{\prime} \in \underline{R}$, as we can see using the definitions. This proves (b).

We shall need the following property:

(c) Let $\rho \in \mathfrak{A}_{\lambda}$ be such that $\sigma_{\rho} \in A_{0}\left(W_{\lambda}\right)$; let $\hat{\rho}$ be the corresponding element of $\hat{\mathfrak{A}}_{\lambda}$. Then there is a unique $w \in \underline{W}_{\lambda}$ such that $w(h)=\hat{\rho}(h)$ for any $h \in \hat{\Pi}_{\lambda}$.

Let $w \in \underline{W}$ be such that $\sigma_{\rho}(t)=w t w^{-1}$ for all $t \in \mathcal{Q}$. (We use 2.3(a).) A case by case check shows that $w$ satisfies the requirement of (c). The uniqueness of $w$ is obvious.

We define a homomorphism $\alpha: \Omega_{\lambda} \rightarrow f A_{\lambda}$ by $z \mapsto \rho$ where $\rho(\chi)=z(\chi)$ for any $\chi \in \Pi_{\lambda}$. We define a homomorphism $\underline{\alpha}: \underline{\Omega}_{\lambda} \rightarrow \mathfrak{A}_{\lambda}$ as the composition $\underline{\Omega}_{\lambda} \rightarrow \Omega_{\lambda} \stackrel{\alpha}{\rightarrow} \mathfrak{A}_{\lambda}$ (the first map is the inclusion (a)). It follows that

(d) image $(\underline{\alpha}) \subset$ image $(\alpha)$.

We define a homomorphism $\tilde{\alpha}: \tilde{\Omega}_{\lambda} \rightarrow \mathfrak{A}_{\lambda}$ as the composition $\tilde{\Omega}_{\lambda} \rightarrow$ $\hat{\mathfrak{A}}_{\lambda} \rightarrow \mathfrak{A}_{\lambda}$ where the first homomorphism is $z \mapsto \rho^{\prime}$ where $\rho^{\prime}(h)=z(h)$ for any $h \in \hat{\Pi}_{\lambda}$ and the second homomorphism is $\hat{\rho} \rightarrow \rho$ for $\rho \in \mathfrak{A}_{\lambda}$. We show that $\alpha=\tilde{\alpha} k$ with $k$ as in (b). Let $w \in \Omega_{\lambda}$. We write $w=t \underline{w}$ where $\underline{w} \in \underline{W}$, $t \in \mathcal{Q}$. We must show that for $\chi \in \Pi_{\lambda}$ we have $\widehat{w(\chi)}=\underline{w}(\hat{\chi})$. This has been verified in the course of proving (b). From $\alpha=\tilde{\alpha} k$ it follows that 
(e) $\operatorname{image}(\alpha) \subset$ image $(\tilde{\alpha})$.

We define $\alpha^{\prime}: A_{0}\left(W_{\lambda}\right) \rightarrow \mathfrak{A}_{\lambda}$ as the composition $A_{0}\left(W_{\lambda}\right) \rightarrow A\left(W_{\lambda}\right) \rightarrow$ $\mathfrak{A}_{\lambda}$ where the first map is the obvious imbedding and the second map is the inverse of the bijection $\rho \mapsto \sigma_{\rho}$. We show:

(f) We have image $(\tilde{\alpha}) \subset \operatorname{image}\left(\alpha^{\prime}\right) \operatorname{image}(\underline{\alpha})$.

Let $z \in \tilde{\Omega}_{\lambda}$. Let $\rho=\tilde{\alpha}(z) \in \mathfrak{A}_{\lambda}$ so that $\hat{\rho}(h)=z(h)$ for any $h \in \hat{\Pi}_{\lambda}$. Let $\sigma=\sigma_{\rho} \in A\left(W_{\lambda}\right)$. By 2.2 we can write $\sigma=\sigma^{\prime} \sigma^{\prime \prime}$ where $\sigma^{\prime} \in A_{0}\left(W_{\lambda}\right)$ and $\sigma^{\prime \prime} \in A\left(W_{\lambda}\right)$ maps $\left\{s_{\chi} ; \chi \in \Pi_{\lambda}-\underline{\Pi}_{\lambda}\right\}$ into itself. Let $\rho^{\prime}, \rho^{\prime \prime}$ in $\mathfrak{A}_{\lambda}$ be such that $\sigma^{\prime}=\sigma_{\rho^{\prime}}, \sigma^{\prime \prime}=\sigma_{\rho^{\prime \prime}}$. Then $\rho=\rho^{\prime} \rho^{\prime \prime}$ and $\rho^{\prime \prime}$ maps $\Pi_{\lambda}-\underline{\Pi}_{\lambda}$ into itself and $\underline{\Pi}_{\lambda}$ into itself. We have $\hat{\rho}=\hat{\rho}^{\prime} \hat{\rho}^{\prime \prime}$ and $\hat{\rho}^{\prime \prime}$ maps $\underline{\Pi}_{\lambda}$ into itself (recall that for $\chi \in \underline{\Pi}_{\lambda}$ we have $\left.\hat{\chi}=\chi\right)$. By (c), we can find $w \in \underline{W}_{\lambda}$ such that $w(h)=\hat{\rho}^{\prime}(h)$ for any $h \in \hat{\Pi}_{\lambda}$. For any $h \in \hat{\Pi}_{\lambda}$ we have $\hat{\rho}^{\prime} \hat{\rho}^{\prime \prime}(h)=z(h)$ hence $w\left(\hat{\rho}^{\prime \prime}(h)\right)=z(h)$, that is $w^{-1} z(h)=\hat{\rho}^{\prime \prime}(h)$. Since $\hat{\rho}^{\prime \prime}\left(\underline{\Pi}_{\lambda}\right)=\underline{\Pi}_{\lambda}$, we deduce that $w^{-1} z\left(\underline{\Pi}_{\lambda}\right)=\underline{\Pi}_{\lambda}$. Since $z \in \underline{W}_{\lambda}^{\prime}, w \in \underline{W}_{\lambda}$, we have $w^{-1} z \in \underline{W}_{\lambda}^{\prime}$. We see that $w^{-1} z \in \underline{\Omega}_{\lambda}$, so that $z=w x$ where $x \in \underline{\Omega}_{\lambda}$. We have $w \in \tilde{\Omega}_{\lambda}, x \in \tilde{\Omega}_{\lambda}$ hence $\rho=\tilde{\alpha}(z)=\tilde{\alpha}(w) \tilde{\alpha}(x)$. We have $\tilde{\alpha}(w) \in \operatorname{image}\left(\alpha^{\prime}\right), \tilde{\alpha}(x) \in \operatorname{image}(\underline{\alpha})$. This proves (f).

We show:

(g) Let $c \in \operatorname{Cell}\left(W_{\lambda}\right)$. The collection of two-sided cells $\left\{z c z^{-1} ; z \in \Omega_{\lambda}\right\}$ coincides with the collection of two-sided cells $\left\{z c z^{-1} ; z \in \underline{\Omega}_{\lambda}\right\}$.

From (e), (f) we deduce that image $(\alpha) \subset$ image $\left(\alpha^{\prime}\right)$ image $(\underline{\alpha})$. Hence, if $z \in \Omega_{\lambda}$ then the automorphism $\omega \mapsto z \omega z^{-1}$ of $W_{\lambda}$ is a product $\sigma^{\prime} \sigma^{\prime \prime}$ where $\sigma^{\prime} \in A_{0}\left(W_{\lambda}\right)$ and $\sigma^{\prime \prime}$ is conjugation by an element $z^{\prime} \in \underline{\Omega}_{\lambda}$. Thus, we have $z c z^{-1}=\sigma^{\prime}\left(z^{\prime} c z^{\prime-1}\right)$. By 2.3(b) we have $\sigma^{\prime}\left(z^{\prime} c z^{\prime-1}\right)=z^{\prime} c z^{\prime-1}$ hence $z c z^{-1}=z^{\prime} c z^{\prime-1}$. We see that the first collection in (g) is contained in the second collection. The reverse containment follows from (a). This proves (g).

2.5. In the remainder of this section we assume that $\mathbf{k}^{\prime}$ has characteristic zero. Let $\mathbf{T}_{f}^{\prime}$ (resp. $\mathbf{k}_{f}^{\prime *}$ ) be the set of elements of finite order in $\mathbf{T}^{\prime}$ (resp. $\left.\mathbf{k}^{\prime *}\right)$. We have canonically $\mathbf{T}^{\prime}=\underline{L}^{\prime} \otimes \mathbf{k}^{\prime *}, \mathbf{T}_{f}^{\prime}=\underline{L}^{\prime} \otimes \mathbf{k}_{f}^{\prime *}$. Recall that in case B we have $\bar{V}_{0}^{\prime}=\underline{V}^{\prime} / \underline{L}^{\prime}$, see 1.3 , and that in case A we write $\bar{V}_{0}^{\prime}=\bar{V}^{\prime}$. We have canonically $\bar{V}_{0}^{\prime}=\underline{L^{\prime}} \otimes(\mathbf{Q} / \mathbf{Z})$. Note that $\underline{W}$ can be viewed as the Weyl group 
of $G$ and that of $G^{\prime}$; it acts naturally on $\mathbf{T}$ and $\mathbf{T}^{\prime}$. We choose an isomorphism $\mathbf{Q} / \mathbf{Z} \stackrel{\sim}{\rightarrow} \mathbf{k}_{f}^{\prime *}$. Via this isomorphism we have $\mathbf{T}_{f}^{\prime}=\underline{L^{\prime}} \otimes \mathbf{Q} / \mathbf{Z}=\bar{V}_{0}^{\prime}$. This is compatible with the $\underline{W}$-actions on $\mathbf{T}^{\prime}$ and $\bar{V}_{0}^{\prime}$. Now let $\mathfrak{o}$ be a $W$-orbit on $\bar{V}^{\prime}$ which is contained in $\bar{V}_{0}^{\prime}$. Note that $\mathfrak{o}$ is in fact a $\underline{W}$-orbit. In case $\mathrm{A}$ this is because $\underline{W}=w$; in case $\mathrm{B}$ this is because the subgroup $\mathcal{Q}$ (see the proof of 1.1(a)) of $W$ acts trivially on $\bar{V}_{0}^{\prime}$. Under the identification $\mathbf{T}_{f}^{\prime}=\bar{V}_{0}^{\prime}$, o can be viewed as a $\underline{W}$-orbit on $\mathbf{T}_{f}$. Let $\lambda \in \mathfrak{o}$. Let $Z(\lambda)$ be the centralizer of $\lambda \in \mathbf{T}^{\prime}$ in $G^{\prime}$ and let $Z(\lambda)^{0}$ be the identity component of $Z(\lambda)$. Since $\lambda$ is semisimple in $G^{\prime}, Z(\lambda)^{0}$ is a reductive group and $\mathbf{T}^{\prime}$ is a maximal torus of it. Let $h \in \underline{R}$. We have $h \in \underline{L}$ so that $h$ can be viewed as a homomorphism $\mathbf{T}^{\prime} \rightarrow K^{*}$. The condition that $h$ is a root of $Z(\lambda)^{0}$ with respect to $\mathbf{T}^{\prime}$ is that $h(\lambda)=1$. An equivalent condition (with $\lambda$ is viewed as an element of $\bar{V}_{0}^{\prime}$ ) is that $(h, \lambda)=0$ or that $h \in \underline{R}_{\lambda}$. We see that the set of roots of $Z(\lambda)^{0}$ is $\underline{R}_{\lambda}$ and the corresponding set of coroots is $\underline{R}_{\lambda}^{\prime} \subset \underline{R}^{\prime}$ (as in 1.2). Using this and 1.3(b), we see that we have $W_{\lambda}=\mathcal{W}\left(Z(\lambda)^{0}\right)$ where $\mathcal{W}\left(Z(\lambda)^{0}\right)$ is the Weyl group (in case A) or the affine Weyl group (in case B) associated to the dual of the reductive group of $Z(\lambda)^{0}$, in the same way as $W$ is the Weyl group (in case A) or the affine Weyl group (in case B) associated to $G$, the dual of $G^{\prime}$. Let $\mathfrak{U}\left(Z(\lambda)^{0}\right)$ be the set of unipotent conjugacy classes of $Z(\lambda)^{0}$; let $\mathfrak{U}_{s p}\left(Z(\lambda)^{0}\right)$ be the set of special unipotent conjugacy classes of $Z(\lambda)^{0}$ (a subset of $\left.\mathfrak{U}\left(Z(\lambda)^{0}\right)\right)$. By $[4,4.8(\mathrm{~b})]$ in case $\mathrm{B}$ we have a canonical bijection

(a) $\mathfrak{U}\left(Z(\lambda)^{0}\right) \leftrightarrow \operatorname{Cell}\left(\mathcal{W}\left(Z(\lambda)^{0}\right)\right)=\operatorname{Cell}\left(W_{\lambda}\right)$;

this restricts to a bijection $\mathfrak{U}_{s p}\left(Z(\lambda)^{0}\right) \leftrightarrow \operatorname{Cell}\left(\underline{W}_{\lambda}\right)$ (each two-sided cell of $\underline{W}_{\lambda}$ is contained in a two-sided cell of $W_{\lambda}$ and this gives an imbedding $\operatorname{Cell}\left(\underline{W}_{l}\right) \subset \operatorname{Cell}\left(W_{\lambda}\right)$. The last bijection can be viewed as a bijection

(b) $\mathfrak{U}_{s p}\left(Z(\lambda)^{0}\right) \leftrightarrow \operatorname{Cell}\left(W_{\lambda}\right)$

in case $\mathrm{A}$.

Now let $\mathfrak{U}(Z(\lambda))$ be the set of unipotent elements of $Z(\lambda)^{0}$ up to $Z(\lambda)$ conjugacy; let $\mathfrak{U}_{s p}(Z(\lambda))$ be the set of special unipotent elements of $Z(\lambda)^{0}$ up to $Z(\lambda)$-conjugacy. Let $N\left(\mathbf{T}^{\prime}\right)$ be the normalizer of $\mathbf{T}^{\prime}$ in $G^{\prime}$. If $w \in \underline{W}_{\lambda}^{\prime}$ and $n \in N\left(\mathbf{T}^{\prime}\right)$ is a representative of $w$ viewed as an element of $N\left(\mathbf{T}^{\prime}\right) / \mathbf{T}^{\prime}$ then $n \in Z(\lambda)$ and $u \mapsto n u n^{-1}$ defines bijections $\mathfrak{U}\left(Z(\lambda)^{0}\right) \rightarrow \mathfrak{U}\left(Z(\lambda)^{0}\right)$, $\mathfrak{U}_{s p}\left(Z(\lambda)^{0}\right) \rightarrow \mathfrak{U}_{s p}\left(Z(\lambda)^{0}\right)$ which depend only on $w$ not on $n$ (since $\mathbf{T}^{\prime} \subset$ $\left.Z(\lambda)^{0}\right)$. This gives an action of $\underline{W}_{\lambda}^{\prime}$ on $\mathfrak{U}\left(Z(\lambda)^{0}\right)$ leaving stable $\mathfrak{U}_{s p}\left(Z(\lambda)^{0}\right)$. (In this action the subgroup $\underline{W}_{\lambda}$ acts trivially.) It is easy to see that two 
unipotent $Z(\lambda)^{0}$-conjugacy classes of $Z(\lambda)^{0}$ are in the same $\underline{W}_{\lambda}^{\prime}$-orbit if and only if they are contained in the same $Z(\lambda)$-conjugacy class. Let $\underline{\Omega}_{\lambda}$ be as in 2.4 (in case B) and let $\underline{\Omega}_{\lambda}=[\lambda, \lambda]$ in case A. Since $\underline{W}_{\lambda}^{\prime}=\underline{\Omega}_{\lambda} \underline{W}_{\lambda}$ it follows that two unipotent $Z(\lambda)^{0}$-conjugacy classes of $Z(\lambda)^{0}$ are in the same $\underline{\Omega}_{\lambda}$-orbit if and only if they are contained in the same $Z(\lambda)$-conjugacy class. Thus we can identify $\mathfrak{U}(Z(\lambda))=\underline{\Omega}_{\lambda} \backslash \mathfrak{U}\left(Z(\lambda)^{0}\right), \mathfrak{U}_{s p}(Z(\lambda))=\underline{\Omega}_{\lambda} \backslash \mathfrak{U}_{s p}\left(Z(\lambda)^{0}\right)$. From the definitions we see that the bijections (a),(b) are compatible with the natural actions of $\underline{W}_{\lambda}^{\prime}$ on the two sides of (a),(b). Taking orbits of these actions we deduce bijections

(c) $\mathfrak{U}(Z(\lambda)) \leftrightarrow \underline{\Omega}_{\lambda} \backslash \operatorname{Cell}\left(W_{\lambda}\right)$ (in case B);

(d) $\mathfrak{U}_{s p}(Z(\lambda)) \leftrightarrow[\lambda, \lambda] \backslash \operatorname{Cell}\left(W_{\lambda}\right)$ (in case $\mathrm{A}$ ).

Now in case B the natural action of $\underline{\Omega}_{\lambda}$ on $\operatorname{Cell}\left(W_{\lambda}\right)$ extends to an action of $[\lambda, \lambda]$ on $\operatorname{Cell}\left(W_{\lambda}\right)$ with the same orbit space (see $2.4(\mathrm{~g})$ ). Thus (c) can be viewed as a bijection

(e) $\mathfrak{U}(Z(\lambda)) \leftrightarrow[\lambda, \lambda] \backslash \operatorname{Cell}\left(W_{\lambda}\right)$ (in case B).

By 1.10 we have a canonical bijection $\operatorname{Cell}(W \times \mathfrak{o}) \leftrightarrow[\lambda, \lambda] \backslash \operatorname{Cell}\left(W_{\lambda}\right)$ (in both cases A,B). Combining with (d),(e) we obtain bijections

$$
\begin{aligned}
& \operatorname{Cell}(W \times \mathfrak{o}) \leftrightarrow \mathfrak{U}(Z(\lambda)) \text { in case B; } \\
& \operatorname{Cell}(W \times \mathfrak{o}) \leftrightarrow \mathfrak{U}_{s p}(Z(\lambda)) \text { in case A. }
\end{aligned}
$$

Using this and the equality $\operatorname{Cell}\left(W \times \bar{V}_{0}^{\prime}\right)=\sqcup_{\mathfrak{o}} \operatorname{Cell}(W \times \mathfrak{o})$ in 1.11 we see that $\operatorname{Cell}\left(W \times \bar{V}_{0}^{\prime}\right)$ is in natural bijection with $\sqcup_{\lambda} \mathfrak{U}(Z(\lambda))$ (in case B) and with $\sqcup_{\lambda} \mathfrak{U}_{s p}(Z(\lambda))$ (in case A) where $\lambda$ runs though a set of representatives for the $\underline{W}$-orbits in $\bar{V}_{0}^{\prime}=\mathbf{T}^{\prime}$. Let $\operatorname{Conj}\left(G^{\prime}\right)$ be the set of conjugacy classes of elements in $G^{\prime}$ with semisimple part of finite order. Let $C o n j_{s p}\left(G^{\prime}\right)$ be the set of conjugacy classes of elements in $G^{\prime}$ with semisimple part of finite order and such that the unipotent part is special in the connected centralizer of the semisimple part. Note that we have canonically $\sqcup_{\lambda} \mathfrak{U}(Z(\lambda))=\operatorname{Conj}\left(G^{\prime}\right)$, $\sqcup_{\lambda} \mathfrak{U}_{s p}(Z(\lambda))=\operatorname{Conj}_{s p}\left(G^{\prime}\right)$. For any $g \in G^{\prime}$ let $\mathcal{B}_{g}^{\prime}$ be the variety of Borel subgroups of $G^{\prime}$ containing $g$. We have the following result.

\section{Theorem 2.6}

(a) We have canonical bijections $\operatorname{Cell}\left(W \times \bar{V}_{0}^{\prime}\right) \leftrightarrow \operatorname{Conj}_{s p}\left(G^{\prime}\right)$ (in case A) and $\operatorname{Cell}\left(W \times \bar{V}_{0}^{\prime}\right) \leftrightarrow \operatorname{Conj}\left(G^{\prime}\right)$ (in case B). 
(b) If $\Delta \in \operatorname{Cell}\left(W \times \bar{V}_{0}^{\prime}\right)$ and $\mathbf{c}$ is the conjugacy class in $G^{\prime}$ corresponding to $\Delta$ under (a), then the value of the a-function (see 1.11) on $\Delta$ is equal to $\operatorname{dim} \mathcal{B}_{g}^{\prime}$ for $g \in \mathbf{c}$.

Now (a) is obtained by combining several statements above; (b) follows from 1.10, 1.11, using [4, 4.8(c)].

\section{References}

1. N. Bourbaki, Groupes et algèbres de Lie, Ch.4,5 et 6, Hermann, Paris, 1968.

2. G. Lusztig, Some examples of square integrable representations of semisimple p-adic groups, Trans. Amer. Math. Soc., 227 (1983), 623-653.

3. G. Lusztig, Characters of reductive groups over a finite field, info Ann. Math. Studies, 107 Princeton U. Press, 1984p.

4. G. Lusztig, Cells in affine Weyl groups, IV, J. Fac. Sci. Univ. Tokyo, 36 (1989), 297-328.

5. G. Lusztig, Monodromic systems on affine flag manifolds, Proc. Royal Soc., 445 (1994), 231-246.

6. G. Lusztig, Hecke algebra with unequal parameters, CRM Monograph Ser. 18, Amer. Math. Soc., 2003.

7. G. Lusztig, Character sheaves on disconnected groups, VI, Represent. Th., 8 (2004), 377-413.

8. G.Lusztig, Character sheaves on disconnected groups,VII, Represent. Th., 9 (2005), 209-266.

9. J. G. M. Mars and T. A. Springer, Character sheaves, Astérisque, 173-174 (1989), 111-198.

10. T. Yokonuma, Sur la structure des anneaux de Hecke d'un groupe de Chevalley fini, C. R. Acad. Sci. Paris Ser. A-B, 264 (1967), A344-A347. 